\title{
New supersymmetric string compactifications
}

\author{
Shamit Kachru \\ Department of Physics and SLAC, Stanford University \\ Stanford, CA 94305/94309, USA \\ E-mail: skachru@stanford.edu
}

\section{Michael B. Schulz}

California Institute of Technology 452-48 Pasadena, CA 91125, USA

E-mail: mschulz@theory.caltech.edu

\section{Prasanta K. Tripathy and Sandip P. Trivedi}

Tata Institute of Fundamental Research

Homi Bhabha Road, Mumbai 400 005, India

E-mail: prasanta@theory.tifr.res.in, sandip@tifr.res.in

ABSTRACT: We describe a new class of supersymmetric string compactifications to $4 \mathrm{~d}$ Minkowski space. These solutions involve type II strings propagating on (orientifolds of) non Calabi-Yau spaces in the presence of background NS and RR fluxes. The simplest examples have descriptions as cosets, generalizing the three-dimensional nilmanifold. They can also be thought of as twisted tori. We derive a formula for the (super)potential governing the light fields, which is generated by the fluxes and certain "twists" in the geometry. Detailed consideration of an example also gives strong evidence that in some cases, these exotic geometries are related by smooth transitions to standard Calabi-Yau or $G_{2}$ compactifications of M-theory.

KEYWORDS: String Duality, Superstring Vacua. 


\section{Contents}

1. Introduction 1

2. Basic formalism 3

2.1 Flux vacua in IIB on $T^{6} / Z_{2}$

2.2 A warm-up: the twisted torus

2.3 Twisted tori in general

3. New geometries from $T^{6} / Z_{2}$

3.1 A detailed example 9

3.2 M-theory limit and the web of vacua 12

3.3 The example as a coset 13

4. Supersymmetry 14

4.1 IIA with $O 4$ planes

4.2 IIB with $O 5$ planes 19

4.3 Superpotential 21

5. Discussion 25

A. T-duality maps of fields

B. T-duality map of spinor conditions 29

\section{Introduction}

The study of Calabi-Yau compactifications [1] has been tremendously fruitful. These models play an important role in deriving realistic models of low-energy particle physics from the heterotic string. Their study has also lead to fascinating discoveries about stringy geometry and mathematical physics [2].

More recently, it has become clear that Calabi-Yau compactifications of type-II strings are also a reasonable starting point for model building. In orientifolds of such models, D-branes wrapping various cycles give rise to non-abelian gauge groups, and can yield a reasonable facsimile of the Standard Model [3]. Background RR and NS fluxes can also be included, and generate a computable (super)potential which stabilizes many of the moduli fields $4-15$. In addition, the fluxes and branes backreact on the bulk geometry, yielding warped compactifications [16, 20, 17. In special cases, the warping can be a large effect [18, 9, 19, 20]. This provides a natural mechanism for explaining hierachies of scales, as suggested by Randall and Sundrum [21]. 
However, such Calabi-Yau orientifolds are merely the tip of the iceberg. One way to see this is as follows. Starting from the type IIB theory on a Calabi-Yau space $M$ in absence of NS three-form flux $\mathcal{H}$, one can construct a dual type-IIA description of the same physics in terms of a mirror Calabi-Yau $W$. On the other hand, in the presence of generic $\mathcal{H}$-flux on $M$, the mirror geometry $W$ can no longer be a Calabi-Yau space. This is clear because mirror symmetry is a kind of generalization of T-duality [22], and T-duality maps nontrivial $\mathcal{H}$ to an interesting deformation of the T-dual metric.

In this way, starting from a solution of the IIB theory on a Calabi-Yau orientifold with background fluxes, one can generate mirror IIA geometries which are not (orientifolds of a) Calabi-Yau, but are supersymmetric solutions of the equations of motion. It should be clear that more generically, one can construct models which are not described by CalabiYau geometries in either picture. For instance, turning on generic NS flux in the IIA non Calabi-Yau geometry will result in solutions whose IIB dual is no longer a Calabi-Yau space.

The resulting models are of interest for phenomenological reasons as well. They are typically expected to have fewer moduli than conventional Calabi-Yau compactifications. In addition, the computable potential on moduli space could, in some cases, be useful from a cosmological viewpoint (for a recent investigation in this direction see [23).

Here, we describe the simplest examples of such new supersymmetric compactifications of type-II strings. Our starting point is the $T^{6} / Z_{2}$ orientifold of type-IIB theory in the presence of NS and RR three-form fluxes, discussed in detail in [12, 13. In simple enough cases these models possess classical isometries, and by performing Buscher duality transformations [24], one is able to construct dual solutions which are not Calabi-Yau geometries. Instead, we find that the dual manifolds are well described as cosets, generalizing the three-dimensional nilmanifold. They can also be thought of as twisted tori, first studied in [25]. The light scalar fields in these models are governed by a (super)potential which is generated by both the fluxes and certain gravitational charges which can be formed from the background metric [26].

This paper concerns itself with type-II strings, but similar questions are also of interest in the context of the heterotic string. Once again, one would like to ask about supersymmetric vacua in the presence of the three-form NS flux [27]. Little is known about such models, but in some cases these heterotic vacua are dual to the type-II ones.

The organization of this paper is as follows. In section [2, we briefly review the class of models studied in [12] and discuss how T-duality in the presence of $\mathcal{H}$-flux generates twisted tori by describing a simple toy example. In section 3 , we derive various dual descriptions of the $T^{6} / Z_{2}$ flux vacua, which exhibit generalizations of the twisting encountered in section 2. For illustration we present a detailed discussion of an $\mathcal{N}=2$ supersymmetric compactification to $4 \mathrm{~d}$ with several dual descriptions involving non Calabi-Yau geometries. We also find that consideration of the M-theory limit indicates that this example can be connected (on the moduli space of vacua) to a smooth type-II Calabi-Yau compactification (with no orientifolding). In section 斗, we directly derive the supersymmetry conditions in the dual pictures, and write down an effective $4 \mathrm{~d}$ superpotential which imposes these constraints. We also verify that this superpotential correctly reproduces expected domain wall tensions, in the spirit of 4,28 . We close with some comments on further directions 
for research in section 5. In two appendices, we summarize the T-duality formulae for the transformations of IIA/IIB fields and spinors which are used throughout the paper.

We end by commenting on some recent related work. The papers [29, 30, 31 discuss flux compactifications and their relation to gauged supergravity. While this work was in progress, we also became aware of several related projects. Work which has significant overlap with the present paper appeared recently in [32, 33]. Heterotic string compactifications on non Calabi-Yau spaces related by duality to the models studied in [12 have appeared in [34] (and earlier work in this direction appeared in [27, 5]). Other compactifications which involve "duality twists" and are related indirectly to the twisted tori which appear in section 2 have appeared in [35, 36]. Finally, some of our results were also found by S. Gukov [37].

\section{Basic formalism}

\subsection{Flux vacua in IIB on $T^{6} / Z_{2}$}

The theories that we will discuss in subsequent sections are all related via T-duality to IIB theory compactified on a $T^{6} / Z_{2}$ orientifold. In this orientifold, the $Z_{2}$ acts to invert all six circles. In the absence of flux, the theory is T-dual to the type-I theory, and preserves $\mathcal{N}=4$ supersymmetry. Since there are 64 O3 planes located at the fixed-points of the $Z_{2}$, RR tadpole cancellation requires that there also be 16 space-filling D3 branes. These are the T-duals of 16 D9 branes of $\mathrm{SO}(32)$ in type-I, and the low energy effective field theory is the same $\mathrm{SO}(32) \mathcal{N}=4$ supersymmetric Yang-Mills theory, coupled to $\mathcal{N}=4$ supergravity, familiar from type-I.

However, this is not the most general solution to the $T^{6} / Z_{2}$ tadpole cancellation condition. This class of compactifications also admits different superselection sectors in which we turn on quantized NS-NS and RR three-form fluxes. Let $\mathcal{H}_{3}$ denote the NS-NS flux and $F_{3}$ denote the RR flux. ${ }^{1}$ These field-strengths satisfy a Dirac quantization condition

$$
\frac{1}{(2 \pi)^{2} \alpha^{\prime}} \int_{\gamma} F_{3}=m_{\gamma} \in \mathbb{Z}, \quad \frac{1}{(2 \pi)^{2} \alpha^{\prime}} \int_{\gamma} \mathcal{H}_{3}=n_{\gamma} \in \mathbb{Z},
$$

where $\gamma$ labels the classes in $H_{3}\left(T^{6}, \mathbb{Z}\right)$. In the presence of such fluxes, the D3 brane charge tadpole cancellation condition reads: ${ }^{2}$

$$
\frac{1}{2} N_{\text {flux }}+N_{D 3}=16
$$

where

$$
N_{\text {flux }}=\frac{1}{(2 \pi)^{4} \alpha^{2}} \int_{T^{6}} \mathcal{H}_{3} \wedge F_{3} .
$$

So if we turn on fluxes, we should in general introduce fewer D3 branes.

\footnotetext{
${ }^{1}$ The calligraphic font on the NS-flux indicates that it is a quantity associated with the IIB orientifold that we are now discussing, and not with one of the T-dual theories that we will describe in later sections. Confusion is less likely for the RR flux, which changes rank under T-duality, so we do not use the calligraphic notation in the RR-sector.

${ }^{2} \mathrm{As}$ in [12], we ignore the possibility of exotic O3 planes. To consistently do this, we must choose the integers in (2.1) to be even, as explained in 13. This will be sufficient for our purposes.
} 
Nonvanishing fluxes give rise to an effective superpotential for the Calabi-Yau complex structure moduli 四. (A detailed derivation of this in the context of IIB orientifolds was given in appendix $\mathrm{A}$ of [9]). The superpotential is

$$
W=\int G_{3} \wedge \Omega
$$

where

$$
G_{3}=F_{3}-\phi \mathcal{H}_{3}
$$

and $\phi$ is the IIB axio-dilaton. It follows that supersymmetric vacua are located at points in complex structure moduli space where $G$ is of type $(2,1)$ and imaginary self-dual. Furthermore, for supersymmetry, the Kähler structure $J$ should be chosen to make $G_{3}$ primitive (i.e. satisfy $J \wedge G_{3}=0$ ). These conditions were studied in detail for the case of $T^{6} / Z_{2}$ in [12], and it was found that for generic choices of the fluxes (2.1) there are no supersymmetric critical points. However, for suitable non-generic choices of flux, one can find vacua with $\mathcal{N}=1,2,3$ supersymmetry (and reduced numbers of moduli).

In the absence of flux, these models admit isometries and various T-dual descriptions exist. The RR three-form flux transforms quite simply under T-duality. Our goal in the following subsections will be to explore what happens when one tries to construct analogous T-dual descriptions in the presence of nontrivial $\mathcal{H}_{3}$-flux.

Before moving on, we should note one further interesting feature of these models. The fluxes and transverse branes and O-planes act as sources for a nontrivial warping of the metric. The 10-metric in string frame takes the form

$$
d s^{2}=e^{-2 A} \eta_{\mu \nu} d x^{\mu} d x^{\nu}+e^{2 A} \tilde{g}_{m n} d x^{m} d x^{n}
$$

where $\mu, \nu=0,1,2,3$ and $m, n=4, \cdots, 9$. The metric $\tilde{g}_{m n}$ is the unwarped metric on the compactification space. The warp-factor $e^{2 A}$ is determined by the equation

$$
-\tilde{\nabla}^{2} e^{4 A}=(2 \pi)^{4}\left(\alpha^{\prime}\right)^{2} g_{s} \tilde{\rho}_{3}+\frac{g_{s}}{12} G_{m n p} \bar{G}^{\widetilde{m p} p}
$$

where tildes denote the use of the unwarped metric, and $\rho_{3}$ refers to the localized D3charge density (which gets contributions from both D3 branes and O3 planes). One can argue as in [9, 13] that under rescaling the metric $\tilde{g}_{m n} \rightarrow \lambda^{2} \tilde{g}_{m n}$, the warp factor behaves like $e^{2 A} \sim 1+O\left(\lambda^{-4}\right)$. Therefore, at large radius and weak coupling, where one trusts the supergravity equations, the corrections due to the warp factor are negligible. In the examples we provide in this paper, one can choose the moduli to lie in a regime where one can neglect the warping.

\subsection{A warm-up: the twisted torus}

As a preliminary indication of what to expect, let us consider the following simple toy model. Imagine starting with a square three-torus $M$ parametrized by $x, y, z$, with metric

$$
d s^{2}=d x^{2}+d y^{2}+d z^{2} .
$$


Suppose one has also turned on $N$ units of NS three-form flux through this torus

$$
\frac{1}{(2 \pi)^{2}\left(\alpha^{\prime}\right)} \int_{M} \mathcal{H}_{3}=N
$$

We can choose a gauge where

$$
\mathcal{B}_{y z}=N x
$$

with other components vanishing. (From now on, we set $(2 \pi)^{2} \alpha^{\prime}=1$ for convenience).

Now, imagine T-dualizing along the $z$ direction. In the T-dual, the $B$ field vanishes. The resulting T-dual metric is

$$
d s^{2}=d x^{2}+d y^{2}+(d z+N x d y)^{2} .
$$

We will call such a space a "twisted torus" or a "nilmanifold," following [38 and [39] respectively. The identifications to be made in interpreting (2.11) are not the same as in a standard $T^{3}$. Instead one should identify ${ }^{3}$

$$
(x, y, z) \cong(x, y+1, z) \cong(x, y, z+1) \cong(x+1, y, z-N y) .
$$

This space has another convenient description, as a coset. Consider $R^{3}$, presented as the space of upper triangular $3 \times 3$ matrices with ones along the diagonal:

$$
g_{N}(x, y, z)=\left(\begin{array}{ccc}
1 & y & -\frac{1}{N} z \\
0 & 1 & x \\
0 & 0 & 1
\end{array}\right)
$$

where $x, y, z$ are real numbers. Let us call this group $\mathcal{G}_{3}^{N}(\mathbb{R})$. For any $N$, this group is isomorphic to the three-dimensional Heisenberg group $\mathcal{H}_{3}$. We can also define $\mathcal{G}_{3}^{N}(\mathbb{Z})$ by considering analogous matrices

$$
g_{N}(a, b, c)=\left(\begin{array}{ccc}
1 & b & -\frac{1}{N} c \\
0 & 1 & a \\
0 & 0 & 1
\end{array}\right)
$$

with $a, b, c$ integers. This has a natural action on $\mathcal{G}_{3}^{N}(\mathbb{R})$ by matrix multiplication. Consider the right-coset $M=\mathcal{G}_{3}^{N}(\mathbb{R}) / \mathcal{G}_{3}^{N}(\mathbb{Z})$. The resulting identifications are

$$
(x, y, z) \sim(x+a, y+b, z-N b y+c) .
$$

A little thought shows that the spaces (2.15) are the same as the twisted tori (2.12).

Since these spaces were obtained as a right-coset, we would naively expect the twisted tori to possess an isometry group corresponding to left-multiplication by $\mathcal{G}_{3}^{N}(\mathbb{R})$. However, the one-forms appearing in the metric (2.11) are the right-invariant forms $\eta^{1}=d x, \eta^{2}=d y$, $\eta^{3}=d z+N x d y$, defined by $g_{N}\left(\eta^{1}, \eta^{2}, \eta^{3}\right)=d g_{N} g_{N}^{-1}(x, y, z) .{ }^{4} \quad$ The would-be Killing

\footnotetext{
${ }^{3}$ The nontrivial identification $(x, y, z) \cong(x+1, y, z-N y)$ is necessary in order that $d z+N x d y=$ $d(z-N y)+N(x+1) d y$ be globally well-defined.

${ }^{4}$ Note that the positive-definite metric 2.11 is not same as the Cartan metric $\operatorname{Tr} d g d g$, which is easily seen to vanish. (Since the group is non-semi-simple, the usual theorem that the Cartan metric is positivedefinite does not apply).
} 
vectors that generate left-multiplication are the right-invariant vector fields $k_{1}=\partial_{x}, k_{2}=$ $\partial_{y}-N x \partial_{z}, k_{3}=\partial_{z}$, dual to the $\eta^{i}$. Of these, only $k_{3}$ is actually an isometry of the metric. ${ }^{5}$ The isometry is the $\mathrm{U}(1)$ of translations in the $z$-direction.

It is easy to see that the twisted torus is topologically distinct from the untwisted torus. For instance, $h^{1}(M)=2$ for $M=$ the twisted $T^{3}$. One can prove this as follows. Since $\mathcal{G}_{3}^{N}(\mathbb{R})$ is topologically $R^{3}$, it follows that $\pi_{1}\left(\mathcal{G}_{3}^{N}(\mathbb{R}) / \mathcal{G}_{3}^{N}(\mathbb{Z})\right)=\mathcal{G}_{3}^{N}(\mathbb{Z})$. The homology group $H_{1}(M, \mathbb{Z})$ is the abelianization of this group, that is, the group $\mathcal{G}_{3}^{N}(\mathbb{Z})$ modulo its commutator subgroup. (Here commutator mean group-commutator $X Y X^{-1} Y^{-1}$, as opposed to algebra-commutator $X Y-Y X)$. It is easy to show that the commutator subgroup is generated by $e^{N}$, where $e=g_{N}(0,0,1)$. The group $H_{1}(\mathbb{Z})$ is then $\mathbb{Z} \times \mathbb{Z} \times \mathbb{Z}_{N}$, with the first factor generated by $g_{N}(1,0,0)$, the second by $g_{N}(0,1,0)$, and the third by $e$ modulo $e^{N}$. The real-valued homology group is $H_{1}(M, \mathbb{R})=\mathbb{R}^{2}$, of dimension two. ${ }^{6}$

Using Cartan's structure equation

$$
d \eta^{a}+\omega^{a}{ }_{b} \wedge \eta^{b}=T^{a}=0, \quad T^{a}=\text { torsion },
$$

it is straightforward to solve for the spin connection of the twisted $T^{3}$. We have

$$
d \eta^{1}=d \eta^{2}=0, \quad d \eta^{3}=N \eta^{1} \wedge \eta^{2}
$$

so the solution is

$$
\omega_{2}^{1}=-\frac{1}{2} N \eta^{3}, \quad \omega^{2}{ }_{3}=\frac{1}{2} N \eta^{1}, \quad \omega^{3}{ }_{1}=\frac{1}{2} N \eta^{2} .
$$

Taking the antisymmetric part of the spin-connection, we obtain a three-form

$$
\omega_{(3)}=\eta^{a} \wedge \eta^{b} \wedge \omega_{a b}=N \eta^{1} \wedge \eta^{2} \wedge \eta^{3} .
$$

This illustrates a general rule. When a component of NS flux is lost through T-duality, it reappears in the antisymmetrized spin-connection as

$$
\omega_{(3)}=k \wedge F_{\mathrm{KK}}+\cdots,
$$

where $k$ is the Killing one-form of the isometry, and $F_{\mathrm{KK}}$ is the flux of the corresponding Kaluza-Klein gauge field [26]. In this case, $A_{\mathrm{KK}}=N x d y, F_{\mathrm{KK}}=N d x \wedge d y$, and $k=$ $g_{z m} d x^{m}=d z+N x d y$.

\footnotetext{
${ }^{5}$ The reader is invited to check that the nonvanishing Lie derivatives of the form $\mathcal{L}_{k} \eta$ are $\mathcal{L}_{k_{1}} \eta^{3}=N d y$ and $\mathcal{L}_{k_{2}} \eta^{3}=-N d x$. Consequently, only $\mathcal{L}_{k_{3}}$ acting on the metric is nonzero.

Another way to understand this is in terms of the generators of right-multiplication. All of these generators are compatible with the metric, but only $k_{3}$ (which has a dual interpretation as generator of either left- or right-multiplication) is compatible with the quotienting that defines the coset. That is, $k_{3}$ is the only generator of right-multiplication that lies in the commutant of $\mathcal{G}^{N}(\mathbb{Z})$ in $\mathcal{G}^{N}(\mathbb{R})$.

${ }^{6}$ The interpretation of the torsion factor $\mathbb{Z}_{N}$ is that that there is a third one-cycle, around which string winding is conserved modulo $N$. This is the T-dual of the statement that momentum on the original $T^{3}$ is conserved modulo $N$ in the presence of $N$ units of NS-flux.
} 
This toy model is not really a solution of the string equations of motion: if one tries to compactify on a $T^{3}$ with $\mathcal{H}_{3}$-flux, both the string coupling and the volume of the torus have tadpoles and want to relax to extreme values. However, in the more elaborate backgrounds reviewed in section 2.1, one can find stable toroidal compactifications with both $\mathcal{H}_{3}$ and $\mathcal{F}$ flux. Then, dualizing along any isometry directions will yield new stable vacua, with "twistings" quite analogous to the one above. These vacua will also have a convenient description as cosets.

One final comment before we move on. Starting with the metric (2.8) and $\mathcal{B}$ field (2.10), we have discussed the geometry which results after one T-duality. It is interesting to ask what happens on doing additional T-dualities. The geometry (2.11) (with $B=0$ ) is independent of the $y$ direction. T-dualizing further along this direction gives rise (using the T-duality rules in appendix (A) to the metric

$$
d s^{2}=\frac{1}{1+N^{2} x^{2}}\left(d z^{2}+d y^{2}\right)+d x^{2}
$$

and $\mathcal{B}$ field

$$
\mathcal{B}_{y z}=\frac{N x}{1+N^{2} x^{2}}
$$

This background looks quite puzzling at first. For example, on going around the $x$ circle the metric of the $z, y$ two-torus, $T_{\{y z\}}^{2}$, is not periodic even up to an $\operatorname{SL}(2, \mathbb{Z})$ transformation (2.21).

A little more thought shows that the metric (2.21) and $\mathcal{B}$ field (2.22) are in fact periodic up to an element of $O(2,2, \mathbb{Z})$ which does not belong to $\mathrm{SL}(2, \mathbb{Z})$. This can be understood as follows. In the metric (2.11), on going around the $x$ circle, the two-torus $T_{\{y z\}}^{2}$ is twisted by an element of $\operatorname{SL}(2, \mathbb{Z})$ which we denote as $A$. T-duality along the $y$ direction, which we denote as $T_{y}$, does not commute with $A$. As a result in the final solution the metric and $\mathcal{B}$ field, (2.21) and (2.22), twist by the transformation $T_{y}^{-1} A T_{y} \in O(2,2, \mathbb{Z})$ on going around the $x$ circle.

The facts above imply that the supergravity approximation is not adequate to describe this background. Momentum modes along the $T_{y z}^{2}$ mix with winding modes on going around the $x$ circle. Since this paper restricts itself to the supergravity approximation, we do not consider backgrounds of this sort any further. We follow a simple rule to avoid them: never T-dualize twice along two directions $a, b$, if $\left[\mathcal{H}_{3}\right]_{a b c} \neq 0$ for any direction $c$ in the starting configuration. Twists by elements of the $O(2,2, \mathbb{Z})$ duality group have been considered in 35,36$]$.

\subsection{Twisted tori in general}

The nilmanifold and related generalizations we discussed above are in fact examples of twisted tori discussed in the seminal paper of Scherk and Schwarz [25.

One way to think about twisted tori in general is as follows (see also [38]): they are parallelizable manifolds with a well defined, nowhere vanishing basis of vielbein fields. Below, we denote this basis of vielbein one-forms as $\eta^{a}, a=1, \ldots, n$. The coordinate basis 
one-forms $d x^{\alpha}$ are related to the vielbein $\eta^{a}$ by

$$
\eta^{a}=\mathrm{U}(x)_{\alpha}^{a} d x^{\alpha}
$$

The quantity $U \in G L(n, \mathbb{R})$ is a matrix which specifies the twisting.

In particular the twisting matrix $U$ must satisfy an important property. The coefficients $f_{b c}^{a}$, defined by

$$
d \eta^{a}=-\frac{1}{2} f_{b c}^{a} \eta^{b} \wedge \eta^{c}
$$

must be constant on a twisted torus. Following [25], we refer to these coefficients as structure constants.

Note that as a result the spin connection is also a constant on a twisted torus. This follows because the spin connection can be expressed in terms of the structure constants as

$$
w_{a b}^{c}=\frac{1}{2}\left(f_{a b}^{c}-\delta_{b s} \delta^{c j} f_{a j}^{s}-\delta^{c j} \delta_{a s} f_{b j}^{s}\right),
$$

(where $\delta^{b s}$ etc denote the Kronecker delta symbol). Finally, it is worth mentioning that in the low-energy theory obtained after KK reduction on a twisted torus, masses for moduli and (non abelian) gauge couplings can arise. These depend only on the structure constants, and have no other depndence on the twisting matrix $U$.

Let us see how this general discussion applies to the examples of section 2.2. Consider the vielbein (2.11)

$$
\left(\begin{array}{c}
\eta^{1} \\
\eta^{2} \\
\eta^{3}
\end{array}\right)=\left(\begin{array}{c}
d x \\
d y \\
d z+N x d y
\end{array}\right) .
$$

Note first that the $\eta^{a}$ are well defined on the twisted torus. In particular as mentioned in the footnote after (2.12), $\eta^{3}$ twists by the appropriate $\mathrm{SL}(2, \mathbb{Z})$ transformation in going around the $x$ circle. The twisting matrix $U$ can be read off from (2.26), and is:

$$
U=\left(\begin{array}{ccc}
1 & 0 & 0 \\
0 & 1 & 0 \\
0 & N x & 1
\end{array}\right)
$$

The structure constants then take the form $f_{12}^{3}=-f_{21}^{3}=-N$, with all other components vanishing. They are indeed constant. ${ }^{7}$ Thus the generalized nilmanifolds of section 2.2 have all the properties of twisted tori discussed in 25.8

There is another way to connect our discussion of twisted tori above with nilmanifolds. The structure constants $f_{b c}^{a}$ in general define a (non-compact) Lie algebra. For the twisted torus of section 2.2 this is the Heisenberg algebra (with $N$ playing the role of $\hbar$ ). The corresponding group is the group of upper triangular matrices $G$. The nilmanifolds are cosets of exactly this group by appropriate discrete subgroups.

\footnotetext{
${ }^{7}$ Strictly speaking, once the effects of warping are included the coefficients $f_{b c}^{a}$ are no longer constant. In this sense the compactification is a generalisation of the twisted torus considered in 25.

${ }^{8}$ There is one more condition on the structure constants in [25], $f_{a b}^{a}=0$. This too is met by the example of section 2.2.
} 


\section{New geometries from $T^{6} / Z_{2}$}

\subsection{A detailed example}

For concreteness, it is helpful to describe explicitly a nontrivial example where one can see the considerations of the previous subsections come into play. As our starting point, we take the $\mathcal{N}=2$ supersymmetric flux compactification on $T^{6} / Z_{2}$ which played an important role in [40]. The fluxes are chosen to be:

$$
\begin{aligned}
F_{3} & =2 d x^{1} \wedge d x^{2} \wedge d y^{3}+2 d y^{1} \wedge d y^{2} \wedge d y^{3} \\
\mathcal{H}_{3} & =2 d x^{1} \wedge d x^{2} \wedge d x^{3}+2 d y^{1} \wedge d y^{2} \wedge d x^{3}
\end{aligned}
$$

(Again, here and in the rest of the paper, we set equal to 1 the factor of $(2 \pi)^{2} \alpha^{\prime}$ that should appear on the r.h.s. when specifying each of the fluxes). The factors of 2 are inserted to avoid various subtleties related to "exotic" O3 planes which arise when the fluxes aren't even. It follows from the equations of 12 that this model has a moduli space of $\mathcal{N}=2$ supersymmetric vacua. Along this moduli space, the $T^{6}$ looks like a $\left(T^{2}\right)^{3}$. The three $T^{2}$ 's lie in the $x^{i} y^{i}$ directions, with $i=1,2,3$, respectively. The complex structure moduli $\tau_{1,2,3}$ of the three two-tori, together with the axio-dilaton $\phi$, satisfy the equations

$$
\begin{aligned}
\phi \tau_{3} & =-1 \\
\tau_{1} \tau_{2} & =-1
\end{aligned}
$$

There are also Kähler moduli which are constrained only by the primitivity condition. For simplicity, we work at a point in moduli space where the metric is diagonal with radii $R_{x^{i}}, R_{y^{i}}$. The constraints on the moduli (3.3) and (3.4) can be written in terms of the radii using $\tau_{i}=i R_{y^{i}} / R_{x^{i}}$.

This is a nongeneric situation; for generic choices of flux, the complex structure and $\phi$ would have been completely frozen. But it is convenient to consider such an example, where the fluxes are quite simple, for several reasons. One is that then the dual geometries are also quite simple. The other, perhaps more important, reason is that then one can argue that the dual description is at large radius (and weak coupling), and hence the dual geometry is meaningful, for an appropriate regime of parameters.

Choose a gauge where

$$
\mathcal{B}_{x^{1} x^{3}}=2 x^{2}, \quad \mathcal{B}_{y^{1} x^{3}}=2 y^{2}
$$

are the nonvanishing components of the $\mathcal{B}_{\mu \nu}$ field on the internal space. It is an easy matter to find various dual geometries now. We want to avoid the subtleties which arise, as described in section 2.2. when one attempts to dualize two directions which appear in a decomposable piece of $H$. We see that while avoiding this, we can still safely perform three T-dualities (along the $x^{1}, y^{1}$ and $y^{3}$ directions) in this model.

Let us make one more comment. In the discussion below, to focus on the essential features, we neglect the effects of the warp factor (2.6), (2.7). As mentioned in section 2 , this is a good approximation for large volume. More complete formulae with the warp factor can be found in section 4 , in the discussion of supersymmetry. 
One T-duality: a IIA dual. There are several choices for the order in which one performs the dualities. It is clear that dualizing the $y^{3}$ circle does not yield any twisting in the geometry of the dual, so we shall save that for our last transformation.

We will obtain a more interesting result by first dualizing along, say, the $x^{1}$ direction. After performing one T-duality along this direction, we obtain a model where the metric now has a nontrivial $2 \times 2$ block in the $x^{1} x^{3}$ coordinates:

$$
d s^{2}=\frac{1}{R_{x^{1}}^{2}}\left(d x^{1}+2 x^{2} d x^{3}\right)^{2}+R_{x^{2}}^{2}\left(d x^{2}\right)^{2}+R_{x^{3}}^{2}\left(d x^{3}\right)^{2}+\sum_{i=1}^{3} R_{y^{i}}^{2}\left(d y^{i}\right)^{2} .
$$

In other words, the $x^{1}, x^{2}, x^{3}$ coordinates are sweeping out the nilmanifold encountered in section 2.2, while the $y^{i}$ still live on a square $T^{3}$.

In this description, one still has a residual $\mathrm{H}_{3}$-field

$$
H_{3}=2 d y^{1} \wedge d y^{2} \wedge d x^{3}, \quad B_{y^{1} x^{3}}=2 y^{2}
$$

along with RR two-form and four-form fluxes

$$
\begin{aligned}
& F_{2}=2 d x^{2} \wedge d y^{3} \\
& F_{4}=2\left(d x^{1}+2 x^{2} d x^{3}\right) \wedge d y^{1} \wedge d y^{2} \wedge d y^{3} .
\end{aligned}
$$

The O3 planes have turned into O4 planes wrapping the $x^{1}$ circle.

The constraints on moduli (3.3), (3.4) can be easily re-written in terms of the new type-IIA variables. They read

$$
\begin{aligned}
\rho_{1} \tau_{2} & =-1 \\
\tilde{R}_{x^{1}} \phi \tau_{3} & =-1
\end{aligned}
$$

where $\rho_{1}$ is the volume modulus $\rho_{1}=i \tilde{R}_{x^{1}} R_{y^{1}}, \tilde{R}_{x^{1}}=1 / R_{x^{1}}$ is the radius of the T-dualized circle in the IIA theory, and all other quantities refer to IIA variables as well. It is clear that by choosing the point in moduli space appropriately, one can make this T-dual description the effective description, as compared to the IIB starting point.

Notice that it follows from appendix A that the piece of the $H$-field which had a leg along the $x^{1}$ direction is encoded after the duality transformation in the spin-connection $\omega$ in the IIA theory. In the notation of appendix $\mathrm{A}$, one finds that

$$
g_{\left(x^{1}\right)}=2 x^{2} d x^{3}, \quad \omega_{\left(x^{1}\right)}=-2 d x^{2} \wedge d x^{3} .
$$

Here, $g_{\left(x^{1}\right)}$ is the Kaluza-Klein gauge-field corresponding to the isometry in the $x^{1}$-direction, and $-\omega_{\left(x^{1}\right)}=d g_{\left(x^{1}\right)}$ is the corresponding field-strength. The Killing one-form corresponding to this isometry is $k=\eta^{1} / R_{x^{1}}^{2}$, where $\eta^{1}=\left(d x^{1}+2 x^{2} d x^{3}\right)$. So, as in section 2.2 and [26], the antisymmetrized spin-connection is

$$
\omega_{(3)}=k \wedge F_{(2)}^{\mathrm{KK}}=\frac{1}{R_{x^{1}}^{2}} d x^{1} \wedge d x^{2} \wedge d x^{3} .
$$


Therefore, explaining the moduli constraints (3.10) and (3.11) directly in the IIA theory should require us to write down a superpotential which has nontrivial dependence on the spin-connection, or equivalently, on the corresponding Kaluza-Klein flux. This expectation will be borne out in section 4 , where we derive the IIA superpotential and show that it can correctly reproduce the constraints (3.10) and (3.11) in this example.

Finally, we note here that this compactification manifold is non-Kähler. One way to prove this is as follows. As in section 2.2, we can use the relationship between the fundamental group and the first homology of the manifold to compute $h^{1}$. In this case, $h^{1}=5$, while for a Kähler manifold $h^{1}$ is always even. As we will describe in section 4.1. this particular example does admit an integrable complex structure, so it is a complex but non-Kähler space.

Two T-dualities: a IIB dual. Again, dualizing the $y^{3}$ coordinate at this stage would not lead to any further interesting twists in the metric. So instead we dualize the $y^{1}$ coordinate. The resulting IIB geometry is characterized by the metric

$$
\begin{aligned}
d s^{2}= & \tilde{R}_{x^{1}}^{2}\left(d x^{1}+2 x^{2} d x^{3}\right)^{2}+R_{x^{2}}\left(d x^{2}\right)^{2}+R_{x^{3}}\left(d x^{3}\right)^{2}+ \\
& +\frac{1}{R_{y^{1}}^{2}}\left(d y^{1}+2 y^{2} d x^{3}\right)^{2}+R_{y^{2}}^{2}\left(d y^{2}\right)^{2}+R_{y^{3}}\left(d y^{3}\right)^{2} .
\end{aligned}
$$

At this stage of the duality, we have reached a state where the $B$-field on the internal space vanishes. However, there is a nontrivial RR three-form flux

$$
F_{3}=2\left(d x^{1}+2 x^{2} d x^{3}\right) \wedge d y^{2} \wedge d y^{3}+2\left(d y^{1}+2 y^{2} d x^{3}\right) \wedge d x^{2} \wedge d y^{3} .
$$

It is simple to T-dualize the moduli constraints (3.3) and (3.4) and one again finds that in a suitable regime of moduli space, this twice T-dualized geometry is the most effective description of the physics. One can again quickly argue that this space is non-Kähler, by writing out the fundamental form $J$ which comes from the metric (3.14). This form has $d J \neq 0$. A more complete discussion of this is given in section 4.2 , where we describe the supersymmetry conditions after two T-dualities.

Three T-dualities: the IIA "mirror". Finally, one can perform a third T-duality along the $y^{3}$ direction. One might call the result a "mirror" geometry to our starting point, since mirror symmetry can be understood as T-duality along supersymmetric $T^{3}$ fibers [22]. The effect of this last duality on the geometry is simply to take the metric from the previous step and flip $R_{y^{3}} \rightarrow 1 / R_{y^{3}}$. There is in addition a nonvanishing $F_{2}$ flux

$$
F_{2}=2\left(d x^{1}+2 x^{2} d x^{3}\right) \wedge d y^{2}+2\left(d y^{1}+2 y^{2} d x^{3}\right) \wedge d x^{2} .
$$

Although this is a mirror description of the original IIB Calabi-Yau orientifold, the resulting metric is not related to a Calabi-Yau metric. The new information is encoded in windings:

- The $x^{1} x^{3} T^{2}$ undergoes an $\mathrm{SL}(2, \mathbb{Z})$ monodromy as one goes around the $x^{2}$ circle.

- The $y^{1} x^{3} T^{2}$ undergoes an $\operatorname{SL}(2, \mathbb{Z})$ monodromy as one goes around the $y^{2}$ direction. 
Altogether, the nontrivial structure of the metric can be encoded in some $\mathrm{SL}(3, \mathbb{Z})$ matrices that act on the $x^{1} y^{1} x^{3}$ directions, as one moves around on the base parametrized by $x^{2} y^{2} y^{3}$. It would be very interesting to find such non Calabi-Yau mirrors of (orientifolds of) more generic Calabi-Yau manifolds with flux. Note that in the example above we Tdualised along the $x^{1}, y^{1}, y^{3}$ direction, so this is the $T^{3}$ fibre of mirror symmetry. The $\mathrm{SL}(3, \mathbb{Z})$ twist then mixes the fibre and base directions of the Calabi-Yau manifold.

After this third T-duality, the moduli constraints become:

$$
\begin{aligned}
& \tilde{R}_{y^{1}} R_{x^{2}}=\tilde{R}_{x^{1}} R_{y^{2}} \\
& \tilde{R}_{y^{1}} \tilde{R}_{x^{1}}=g_{s} R_{x^{3}}
\end{aligned}
$$

where now all variables appearing are the appropriate variables for the final IIA description (each $\tilde{R}$ is the inverse of the original starting IIA radius, and $g_{s}$ is the final IIA coupling). By choosing an appropriate regime of couplings and radii, one can satisfy these constraints while making the description after three T-dualities the most effective description.

\subsection{M-theory limit and the web of vacua}

Our final IIA background has the following property. ${ }^{9}$ By taking the strong-coupling limit of this IIA vacuum one can get an M-theory description where all radii (including that of the 11th dimension) are large in 11d Planck units, consistent with the constraints (3.17) and (3.18). But since the IIA background was characterized by only a nontrivial metric and $F_{2}$, the M-theory description should be purely geometrical.

To obtain $4 \mathrm{~d} \mathcal{N}=2$ supersymmetry from M-theory, one should compactify it on a Calabi-Yau threefold times a circle. Therefore, the 11d limit of this model should yield Mtheory on some Calabi-Yau threefold $X$ times a circle. From this viewpoint, we have done a twisted reduction (presumably using a twisted circle in the $T^{3}$-fibers of the Calabi-Yau threefold) to get our IIA model. It may be possible to characterize this threefold more concretely, using facts about the M-theory lift of the D6 branes and O6 planes. Since it is known how to write the M-theory lift of O6 planes in terms of the Atiyah-Hitchin space, and how to relate D6 branes to ALE metrics in M-theory [11, the construction of the Calabi-Yau $X$ should involve gluing these local features together into a compact geometry. In fact, one can obtain a family of models with different numbers of D6 branes (and different twisting) by rescaling the original fluxes. In particular, it is possible to rescale the fluxes so that one satisfies the RR tadpole conditions with no D6 branes. The M-theory lift of this model would only involve a gluing of Atiyah-Hitchin spaces (and the right twisting of the $S^{1}$ to give rise to $F_{2}$ ) in the M-theory limit. It would be interesting to more completely characterize the Calabi-Yau manifolds one gets by lifting this set of models.

Even without detailed knowledge of the structure of $X$, this result is interesting because it implies that the orientifold example we have described here is connected, on the moduli space of vacua, to type-IIA compactifications on smooth Calabi-Yau threefolds (with no orientifolding). It was already shown in [40] that the model with no fluxes, which is just dual to the standard $\mathcal{N}=4$ supersymmetric compactification of the heterotic string on $T^{6}$,

\footnotetext{
${ }^{9}$ We thank P. Berglund and N. Warner for emphasizing this point to us.
} 
is connected by vacuum bubbles (of very low tension, in appropriate regimes) to the other flux vacua on $T^{6} / Z_{2}$. In particular, our $\mathcal{N}=2$ model lies on the same configuration space as the heterotic string on $T^{6}$ (or type-II on $K 3 \times T^{2}$ ), in a meaningful sense. But this, combined with our present result, implies that the web of $\mathcal{N}=2$ type-II compactifications on Calabi-Yau spaces is connected (slightly "off" the moduli space) to the $\mathcal{N}=4$ models, and to the $\mathcal{N}=1,2$ flux models of [12].

Finally, it should be clear that we have considered this $\mathcal{N}=2$ model for its simplicity, and because we are confident that vacua with extended supersymmetry exist as solutions to the full theory. However, our considerations could be repeated with many $\mathcal{N}=1$ flux vacua (in the approximation that the flux-generated potential is the full potential). In suitable examples, where there is a IIA dual picture involving only $F_{2}$ flux, one would then expect to find a connection between these flux models and M-theory compactifications on manifolds of $G_{2}$ holonomy.

\subsection{The example as a coset}

The example described above can also be characterized simply as a coset, in analogy with the nilmanifold of section 2.2. Although we will discuss the procedure which allows us to do this in our example, it should be clear that the ideas would generalize to other "twisted tori." For simplicity, we will provide the coset description of the 5-manifold $Y$ spanned by $\left(x^{1}, x^{2}, x^{3}, y^{1}, y^{2}\right)$ in (3.14) (the $y^{3}$ direction is just an extra circle in the geometry), with all radii set to 1 .

A convenient basis of invariant one-forms on $Y$ is provided by

$$
\begin{array}{ll}
\eta^{1}=d x^{1}+2 x^{2} d x^{3}, & \eta^{2}=d x^{2}, \\
\eta^{4}=d y^{1}+2 y^{2} d x^{3}, & \eta^{5}=d y^{2} .
\end{array}
$$

These satisfy an equation of the form

$$
d \eta^{i}=-\frac{1}{2} f_{j k}^{i} \eta^{j} \wedge \eta^{k}
$$

where $f$ is antisymmetric on its two lower indices. The only non-vanishing components of $f$ in this case are

$$
f_{32}^{1}=-f_{23}^{1}=2, \quad f_{35}^{4}=-f_{53}^{4}=2 .
$$

Given such "structure constants" $f_{j k}^{i}$, we can naturally write down a Lie algebra with generators $E_{i}$, satisfying the commutation relations:

$$
\left[E_{j}, E_{k}\right]=f_{j k}^{i} E_{i} .
$$

A simple matrix representation of generators satisfying these relations can be written down as follows. Define the matrices $\mathcal{E}_{\alpha \beta}$ which have only one nonzero entry:

$$
\left(\mathcal{E}_{\alpha \beta}\right)_{i j}=\delta_{\alpha, i} \delta_{\beta, j} .
$$

Then a representation of the algebra (3.22) with structure constants (3.21) is given by choosing

$$
E_{1}=2 \mathcal{E}_{24}, \quad E_{2}=2 \mathcal{E}_{23}, \quad E_{3}=-2 \mathcal{E}_{34}, \quad E_{4}=2 \mathcal{E}_{14}, \quad E_{5}=2 \mathcal{E}_{13} .
$$


It is easy to check that these generators have the property that at the quadratic level, all products vanish except

$$
E_{2} E_{3}=-2 E_{1}, \quad E_{5} E_{3}=-2 E_{4} .
$$

Therefore, when we exponentiate the Lie algebra to form a group $G$, we find that one can write the generic group element as

$$
g=\mathbf{1}+x_{1} E_{1}+x_{2} E_{2}+x_{3} E_{3}+y_{1} E_{4}+y_{2} E_{5} .
$$

Here $\mathbf{1}$ is the unit matrix and the $x$ s and $y$ s are real numbers. We can also define a group $G(\mathbb{Z})$, as the group whose elements can be written as

$$
h=\mathbf{1}+\sum_{i=1}^{5} n_{i} E_{i}
$$

with $n_{i} \in \mathbb{Z}$.

Now, consider identifying points in $G$ under left-multiplication by elements of $G(\mathbb{Z})$. One sees that

$$
\begin{aligned}
(\mathbf{1}+ & \left.n_{i} E_{i}\right)\left(\mathbf{1}+x_{1} E_{1}+x_{2} E_{2}+x_{3} E_{3}+y_{1} E_{4}+y_{2} E_{5}\right)= \\
= & \mathbf{1}+E_{1}\left(n_{1}+x_{1}-2 n_{2} x_{3}\right)+E_{2}\left(n_{2}+x_{2}\right)+E_{3}\left(n_{3}+x_{3}\right)+ \\
& +E_{4}\left(n_{4}+y_{1}-2 n_{5} x_{3}\right)+E_{5}\left(n_{5}+y_{2}\right) .
\end{aligned}
$$

Therefore, the identifications generated by the $G(\mathbb{Z})$ action are precisely those which are characteristic of the twisted geometry with metric (3.14). In particular, the twisted identifications

$$
\left(x^{1}, x^{2}, x^{3}, y^{1}, y^{2}\right) \sim\left(x^{1}-2 x^{3}, x^{2}+1, x^{3}, y^{1}, y^{2}\right) \sim\left(x^{1}, x^{2}, x^{3}, y^{1}-2 x^{3}, y^{2}+1\right)
$$

are reproduced by (3.28).

The structure described here generalizes to the full set of T-duals of flux vacua on $T^{6} / Z_{2}$. The invariant one-forms in the twisted picture always define structure constants via an equation of the form (3.20). These structure constants can be used to define a Lie algebra and a corresponding Lie group $G$, which admits a simple representation in terms of upper triangular matrices. The resulting generalized nilmanifold geometry is a coset of $G$ by the appropriate discrete subgroup.

\section{Supersymmetry}

In this section we analyse the spinor conditions and determine the requirements imposed by $\mathcal{N}=1$ supersymmetry in the various T-dual descriptions. Starting from the IIB backgrounds in the class described in section 2.1, we characterize the theories obtained after one T-duality in section 4.1 and those obtained after two T-dualities in section 4.2 . The supersymmetry requirements will also fix the superpotential (the analogue of (2.4) in the dual theories, as is discussed in section 4.3 . 
Our approach is straightforward. In the starting IIB theory the neccessary and sufficient conditions for susy in the presence of flux are well know [12, 11], and were summarized in section 2.1. We use the known rules of T-duality [43, 44, summarized in Apps. A and $\mathrm{B}$, to map these conditions to the dual theories. This yields the required conditions for susy in the dual variables.

\subsection{IIA with $O 4$ planes}

We start with the IIB theory with $O 3$ planes. In the discussion below, we will follow the notation of [42] closely, see also [12]. We consider the conditions imposed by supersymmetry on the metric and flux in the original IIB theory, and map them to conditions in the IIA theory.

The supersymmetry conditions. Our notation is as follows. We denote the direction along which T-duality is carried out as $x . \mu, \nu$ refer to coordinate indices along all six internal directions, while $\alpha, \beta$ refer to coordinate indices along the five internal directions, not including $x$. Finally, $a, b$ denote the six tangent space indices. One more comment before we get started. Unlike much of the rest of this paper, we include the warp factor in this subsection.

Supersymmetry requires that metric of the starting IIB theory is conformally $C Y_{3}$ (more generally it is a manifold with reduced holonomy, in our case $\left.T^{6}\right) \cdot{ }^{10}$ Up to the warp factor it is therefore complex and Kähler. Let

$$
\tilde{\epsilon}=\tilde{\epsilon}_{L}+i \tilde{\epsilon}_{R}
$$

denote the spinor in the IIB theory which meets the requirements of supersymmetry.

As discussed in [11, 42], $\tilde{\epsilon}$ is of B-type and therefore has definite $\mathrm{SO}(6)$ chirality. An almost complex structure (ACS) can then be defined by

$$
J_{b}^{a}=\tilde{\epsilon}^{\dagger}\left[\gamma^{a}, \gamma_{b}\right] \tilde{\epsilon}
$$

(we take $\tilde{\epsilon}$ to be appropriately normalised so that $J^{2}=-1$ ). For $T^{6} / Z_{2}$ the spinor $\tilde{\epsilon}$ is a constant independent of the internal coordinates, and it is easy to see that the Nijenhuis tensor vanishes. As a result the ACS (4.2) is integrable.

Now we turn to the metric in the IIA theory obtained by one T-duality. We denote the direction along which the T-duality is carried out as $x$. We start with the metric on the $T^{6} / Z_{2}$ in IIB

$$
d s^{2}=e^{2 A} j_{\mu \nu} d x^{\mu} d x^{\nu}
$$

where $j_{\mu \nu}$ is constant and $A$ is the warp factor. There is also a $\mathcal{B}$ field: ${ }^{11}$

$$
\left[\mathcal{B}_{2}\right]_{x \alpha}=\left[\mathcal{H}_{3}\right]_{x \alpha \beta} x^{\beta} .
$$

\footnotetext{
${ }^{10}$ Here we only consider compactifications where the dilaton is a constant.

${ }^{11}$ Other components of the $\mathcal{B}$ field do not enter in determining the dual metric.
} 
We find after using the rules for T-duality, appendix A, that the resulting metric in IIA can be written as

$$
d s^{2}=e^{-2 A} \frac{1}{j_{x x}} \eta^{x} \eta^{x}+e^{2 A}\left(j_{\alpha \beta}-\frac{j_{\alpha x} j_{\beta x}}{j_{x x}}\right) \eta^{\alpha} \eta^{\beta} .
$$

Here, $\left\{\eta^{x}, \eta^{\alpha}\right\}, \alpha=2, \ldots, 6$, is a basis of one-forms defined by

$$
\eta^{x}=d x-\mathcal{B}_{x \alpha} d x^{\alpha}
$$

and

$$
\eta^{\alpha}=d x^{\alpha}, \quad \alpha=2, \ldots, 6 .
$$

Using $\mathcal{B}_{x \alpha}=-g_{x \alpha} / g_{x x}$ (from appendix A), we can equivalently write (4.6) as

$$
g_{x x} \eta^{x}=k_{(x)} \equiv g_{x \mu} d x^{\mu} .
$$

The quantitity $k_{(x)}$ in eq. (4.8) is the Killing one-form. It is one-form that is dual to the Killing vector $\partial_{x}$, which generates translations in the $x$-direction. (In components, $k_{(x)}^{\mu}=\delta_{x}^{\mu}$, so $\left.k_{(x) \mu}=g_{x \mu}\right)$.

A nowhere vanishing, globally well-defined vielbein field, $\hat{\eta}^{a}, a=1, \ldots, 6$, can be defined in terms of the $\eta^{x}, \eta^{\alpha}$ by diagonalizing the metric (4.5). This is in accord with section 2.3 where we had discussed that twisted tori are in general parallelizable manifolds.

Next we turn to the spinor analysis. The spinor transformations rules under T-duality show, generally, that if $\tilde{\epsilon}$ (4.1) meets the spinor conditions for susy in IIB, then the spinor

$$
\epsilon=\epsilon_{L}+i \epsilon_{R}
$$

meets the required conditions in the IIA theory. Here $\epsilon$ is defined in terms of the IIB spinor as follows:

$$
\tilde{\epsilon}_{L}=\Omega \epsilon_{L}, \quad \tilde{\epsilon}_{R}=\epsilon_{R}
$$

$\Omega$ is defined in appendix $\mathrm{B}$.

Consider now the following definition of an ACS in the IIA case. It is given by the same matrix $J_{b}^{a}$ defined in (4.2), but with the indices $a, b$ taking values in the $\hat{\eta}^{a}$ tangent space basis (defined after (4.7)) of the dual manifold. Expressed in terms of the IIA spniors the almost complex structure takes the form:

$$
J_{b}^{a}=\left(\Omega \epsilon_{L}+i \epsilon_{R}\right)^{\dagger}\left[\gamma^{a}, \gamma_{b}\right]\left(\Omega \epsilon_{L}+i \epsilon_{R}\right) .
$$

It is useful to understand this ACS in the basis of one-forms defined in (4.6), (4.7). Group the $\eta^{x}, \eta^{\alpha}$ into two sets, $\eta^{1}=\eta^{x}, \eta^{2}, \eta^{3}$ and $\eta^{4}, \eta^{5}, \eta^{6}$. Then define holomorphic one forms as

$$
\eta_{H}^{i}=\eta^{i}+\tau_{j}^{i} \eta^{3+j}, \quad i, j=1, \ldots 3 .
$$

This choice of holomorphic one forms defines an almost complex structure (ACS) parametrized by the matrix $\tau_{j}^{i}$. The reader will notice that the above steps are analogous to parametrizing the complex structure for a torus. Keeping that analogy in mind we 
will refer to $\tau_{j}^{i}$ as the period matrix of the twisted torus below. Since the spinor $\tilde{\epsilon}$ (4.1) is a constant, the ACS defined in (4.11) is a member of this family, and corresponds to a particular choice of the matrix $\tau_{j}^{i}$.

In the basis of holomorphic one-forms the metric (4.5) can be written as

$$
d s^{2}=g_{i \bar{\jmath}} \eta_{H}^{i} \bar{\eta}_{H}^{\bar{\jmath}}
$$

where $g_{i \bar{\jmath}}$ can be easily obtained in terms of the IIA metric (4.5). This shows that the metric is hermitean with respect to the ACS (4.11). We can now define a two-form, called the fundamental two-form,

$$
J_{I I A}=i g_{i \bar{\jmath}} \eta_{H}^{i} \wedge \bar{\eta}_{H}^{\bar{\jmath}} .
$$

We will see towards the end of this section that the ACS defined above is not integrable. One can also show that the fundamental two-form, (4.14), is not closed. ${ }^{12}$ This implies that the dual geometry is not Kähler. We will see below that an integrable ACS can be defined in the IIA theory, but even with this choice, the fundamental two-form is not closed and the manifold is not Kähler. This is in agreement with our discussion of the specific example in section 3.1, where we showed that the first homology group of the dual manifold is odd dimensional.

The main motivation for considering the ACS above is that the resulting susy conditions on the flux are easy to state, as we shall now see. Consider first the requirements imposed by susy on the flux in the IIB theory. These are that the $G_{3}$ flux should be of type $(2,1)$ and primitive, as reviewed in section 2.1. We now map these conditions to the IIA side.

As discussed in appendix $\mathrm{B}$, the flux $G_{3}$ maps on the IIA side to the three-form

$$
G_{\text {IIA }}=\left(\tilde{F}_{4(x)}+g_{x x} \eta^{x} \wedge F_{2}\right)-i\left(\frac{\sqrt{g_{x x}}}{g_{s}^{\text {IIA }}}\right)\left(H_{3}-g_{x x} \eta^{x} \wedge \omega_{(x)}\right) .
$$

Eq. (4.15) is based notation that is also introduced in appendix B, we will comment more on the various terms that appear in it below.

With the choice of ACS specified above in (4.11) and (4.9), it then follows, as we illustrate in appendix $\mathrm{B}$, that the $(2,1)$ and primitivity conditions map to two requirements on the IIA side. First, that $G_{\text {IIA }}$ is of type $(2,1)$ with respect to the ACS (4.11), (4.9). And second, that it satisfies the condition:

$$
J_{\text {IIA }} \wedge G_{\text {IIA }}=0,
$$

where $J_{\text {IIA }}$ is the fundamental two-form (4.14) in the IIA theory. We will refer to (4.16) as the primitivity condition, since it is analogous to the corresponding condition in the IIB theory.

Before going further let us discuss the various terms in (4.15) in more detail. These arise from the flux $G_{3}$, (2.5), after T-duality. $\tilde{F}_{4(x)}$ in (4.15), stands for the gauge invariant

\footnotetext{
${ }^{12}$ For example, one term in $d J$ arises from, $d \eta_{H}^{1}=d \eta^{x} \neq 0$. Another term arises because $g_{i \bar{\jmath}}$ depends on the warp factor, $A$, (4.3).
} 
four-form in IIA with one leg along the $x$ direction. It arises from $F_{3}$ in the IIB theory with no leg in the $x$ direction. Similarly, $F_{2}$ and $H_{3}$ denote the two form and NS three form in IIA, these terms arise from $F_{3}$ with a leg along the $x$ direction and from $\mathcal{H}_{3}$ without a leg along the $x$ direction respectively. The quantity $\sqrt{g_{x x}} / g_{s}^{\mathrm{IIA}}$ is simply the dilaton in the IIB theory. Finally, the last term in (4.15), arise by T-duality from $\mathcal{H}_{3}$ with a leg along the $x$ direction. The quantity $k_{(x)}=g_{x x} \eta^{x}$ is the Killing one-form (4.8) associated with the isometry in the $x$-direction, and $-\omega_{(x)}$ is the corresponding Kaluza-Klein field-strength. Together, $-g_{x x} \eta^{x} \wedge \omega_{(x)}$ is the antisymmetrised spin-connection three-form $\omega_{(3)},{ }^{13}$ in accord with our discussion in section 22, (2.20).

It is also worth commenting that from a IIA perspective, forgetting about the original IIB description, the direction $x$ is singled out in (4.15) (and in the IIA superpotential) because the $O 4$ planes extend along it.

Below we comment in more detail on the ACS defined above in the susy analysis. In section 4.3, we discuss how to formulate a superpotential in the IIA theory which implements the $(2,1)$ condition on the flux, $G_{I I A}$.

The ACS in more detail. It is clear that the ACS defined in the IIA theory above, (4.11), bears a close relation to the complex structure in the starting IIB theory, (4.2). However, in the IIA theory, due to the non-trivially twisted torus, the ACS is not integrable, in general. The condition for integrability [45] is easy to state in terms of the structure constants which specify the twist, (2.24). In the holomorphic basis (4.12) (with $i, \bar{\imath}$ denoting holomorphic and antiholomorphic indices respectively), it takes the form:

$$
f_{\bar{\jmath} \bar{k}}^{i}=f_{j k}^{\bar{\imath}}=0 .
$$

The lack of integrability is perhaps best illustrated with an example. Consider the example of one T-duality discussed in section 3.1. We start in the IIB theory at a point in moduli space where the $T^{6}$ is of the form $\left(T^{2}\right)^{3}$, with the modular parameters of the three $T^{2}$ s being purely imaginary, of the form $\tau_{i}=i R_{y^{i}} / R_{x^{i}}$. The three holomorphic differentials then are $d z^{i}=d x^{i}+\tau_{i} d y^{i}$. For simplicity we also take the metric to be of form: $d s^{2}=d z^{i} d \bar{z}^{\bar{\imath}}$.

In the IIA dual theory the resulting ACS (4.11) corresponds to the choice of the period matrix $\tau_{j}^{i}$, (4.12), of the form

$$
\tau_{j}^{i}=\operatorname{diag}\left(\tau_{1}, \tau_{2}, \tau_{3}\right) .
$$

The three holomorphic one forms are then given by

$$
\eta_{H}^{1}=\eta^{x}+\tau_{1} \eta^{4}, \quad \eta_{H}^{2}=\eta^{2}+\tau_{2} \eta^{5}, \quad \eta_{H}^{3}=\eta^{3}+\tau_{3} \eta^{6} .
$$

We will skip some of the details here, but this can be established by using the matrix $Q_{\nu}^{\mu}$ defined in appendix $\mathrm{B}$, to relate the vielbein $\hat{\eta}^{a}$, which appears in (4.11), to the basis (4.6), (4.7), which in turn is related to the holomorphic one forms in (4.12).

\footnotetext{
${ }^{13}$ All other components of the antisymmetrized spin-connection are required to vanish by the orientifold projection.
} 
To test for integrability, we consider

$$
d \eta_{H}^{1}=d \eta^{x}=\eta^{2} \wedge \eta^{3} .
$$

Expressing the r.h.s. in terms of the holomorphic basis (4.19), one sees that $f \frac{1}{\overline{2} 3} \neq 0$, so 4.17 ) is not met. ${ }^{14}$

We have not explored in general whether an integrable ACS can be defined in the dual model. For the example considered above the answer turns out to be yes. E.g., consider an alternate choice of holomorphic one forms

$$
\eta_{H}^{1}=\eta^{1}+\tau_{1} \eta^{4}, \quad \eta_{H}^{2}=\eta^{2}+\tau_{2} \eta^{3}, \quad \eta_{H}^{3}=\eta^{5}+\tau_{3} \eta^{6} .
$$

Then one can show that $d \eta_{H}^{1} \sim \eta_{H}^{2} \wedge \bar{\eta}_{H}^{2}$, so (4.17) is now met.

\subsection{IIB with $O 5$ planes}

We turn next to the model obtained by two T-dualities. Since the analysis is very similar to the one T-duality case, we will be brief below. ${ }^{15}$ The two directions along which T-duality is done are denoted by $x, y$. Using the rules in appendix $\mathrm{A}$ the metric after two T-dualities can be written as

$$
d s^{2}=\hat{j}_{x x} \eta^{x} \eta^{x}+\hat{j}_{y y} \eta^{y} \eta^{y}+2 \hat{j}_{x y} \eta^{x} \eta^{y}+\hat{j}_{\hat{\alpha} \hat{\beta}} \eta^{\hat{\alpha}} \eta^{\hat{\beta}} .
$$

Here $\eta^{x}, \eta^{y}, \eta^{\hat{\alpha}}$ denote a basis of one-forms given by:

$$
\begin{aligned}
& \eta^{x}=d x-\mathcal{B}_{x \hat{\alpha}} d x^{\hat{\alpha}}=d x+\frac{\hat{j}_{y y}}{\operatorname{det}_{x y} \hat{j}}\left(\hat{j}_{x x} \hat{j}_{(x)}-\hat{j}_{x y} \hat{j}_{(y)}\right), \\
& \eta^{y}=d y-\mathcal{B}_{y \hat{\alpha}} d x^{\hat{\alpha}}=d y+\frac{\hat{j}_{x x}}{\operatorname{det}_{x y} \hat{j}}\left(\hat{j}_{y y} \hat{j}_{(y)}-\hat{j}_{x y} \hat{j}_{(x)}\right)
\end{aligned}
$$

and

$$
\eta^{\hat{\alpha}}=d x^{\hat{\alpha}} .
$$

The notation used above is as follows. The metric components above, which are denoted with a hat superscript, are constant. We have also used the $\operatorname{definitions} \operatorname{det}_{x y} \hat{j} \equiv\left(\hat{j}_{x x} \hat{j}_{y y}-\right.$ $\left.\hat{j}_{x y}^{2}\right)$ and $\hat{j}_{(x)} \equiv \hat{j}_{x \hat{\alpha}} d x^{\hat{\alpha}} / \hat{j}_{x x}, \hat{j}_{(y)} \equiv \hat{j}_{y \hat{\alpha}} d x^{\hat{\alpha}} / \hat{j}_{y y}$. Finally, the indices $\hat{\alpha}, \hat{\beta}$ run over all compact direction except $x, y$.

The analog of eq. (4.8) is

$$
\begin{aligned}
& \hat{j}_{x x} \eta^{x}+\hat{j}_{x y} \eta^{y}=\hat{k}_{(x)} \equiv \hat{j}_{x \mu} d x^{\mu}, \\
& \hat{j}_{y x} \eta^{x}+\hat{j}_{y y} \eta^{y}=\hat{k}_{(y)} \equiv \hat{j}_{y \mu} d x^{\mu} .
\end{aligned}
$$

Here, $\hat{k}_{(x)}$ and $\hat{k}_{(y)}$ are the Killing one-forms that are dual to the Killing vectors $\partial_{x}$ and $\partial_{y}$, which generate translations in the $x$ - and $y$-directions, respectively. (The determinants in the definition of $\eta^{x}, \eta^{y}$ cancel out in the linear combinations on the l.h.s. of (4.26), leaving the r.h.s.).

\footnotetext{
${ }^{14}$ We have neglected the effects of warping here. Incorporating them does not change the conclusions. $\eta_{H}^{1}$ is now given by $\eta_{H}^{1}=e^{-A} \eta^{x}+\tau_{1} e^{A} \eta^{4}$, so that $d \eta_{H}^{1}$ has an additional term.

${ }^{15}$ We neglect the warp factor here, this can be included along the lines of the previous section.
} 
Once again we define an ACS in the T-dual theory, in terms of the spinor $\hat{\tilde{\epsilon}}$. In a vielbein basis $\hat{\eta}^{a}$ which is obtained by diagonalising the metric above, it takes the form (4.2). Holomorphic one forms analogous to (4.12) can now be defined, and the metric (4.22) can be written in the form (4.13) in this basis. This allows us to define a fundamental two-form. It takes the form:

$$
\hat{J}=i \hat{j}_{i \bar{j}} \eta_{H}^{i} \wedge \eta_{H}^{\bar{j}}
$$

As discussed in appendix $\mathbb{B}$, the $G_{3}$ flux of the starting IIB theory now maps to a three form

$$
\begin{aligned}
\hat{G}_{3}=\left\{\hat{\tilde{F}}_{5(y x)}+\left(\hat{j}_{x x} \eta^{x}+\hat{j}_{x y} \eta^{y}\right) \wedge \hat{\tilde{F}}_{3(y)}-\left(\hat{j}_{x y} \eta^{x}+\hat{j}_{y y} \eta^{y}\right) \wedge \hat{\tilde{F}}_{3(x)}+\right. \\
\left.+\left(\operatorname{det}_{x y} \hat{j}\right) \eta^{x} \wedge \eta^{y} \wedge \hat{F}_{1}\right\}-\left(\frac{i}{\hat{g}_{s}^{\mathrm{IIB}}}\right) \sqrt{\operatorname{det}_{x y} \hat{j}}\left\{\hat{\mathcal{H}}_{3}+\hat{j}_{x x} \eta^{x} \wedge d \hat{j}_{(x)}+\hat{j}_{y y} \eta^{y} \wedge d \hat{j}_{(y)}\right\}
\end{aligned}
$$

Our notation is defined in appendix B. Supersymmetry then leads to the conditions that $\hat{G}_{3}$ is of type $(2,1)$ (with reference to the ACS defined above) and primitive (with respect to the fundamental form (4.27)).

Let us comment briefly on the various terms in (4.28). These arise from $G_{3},(2.5)$, in the starting IIB theory after T-duality. $\hat{\tilde{F}}_{5(y x)}$, arises from $\tilde{F}_{3}$ with no leg along the $x, y$ directions, $\hat{\tilde{F}}_{3(x)}, \hat{\tilde{F}}_{3(y)}$, from $\tilde{F}_{3}$ with a leg along either $y$ or $x$ direction and $\hat{F}$ from $\tilde{F}_{3}$ with a leg along both $x, y$ directions. $\hat{\mathcal{H}}_{3}$ arises from $\mathcal{H}_{3}$ with no leg along the $x, y$ directions. Finally, the last two terms in (4.28), are components of the antisymmetrised spin connection in the dual theory. They arise from $\mathcal{H}_{3}$ with one leg along the $x$ or $y$ directions. In this context it is worth noting that the last two terms in (1.28) can be rewritten as:

$$
\hat{j}_{x x} \eta^{x} \wedge d \hat{j}_{(x)}+\hat{j}_{y y} \eta^{y} \wedge d \hat{j}_{(y)}=\hat{k}_{(x)} \wedge F^{x}+\hat{k}_{(y)} \wedge F^{y},
$$

where $\hat{k}_{(x, y)}$ are the Killing one forms defined in 4.26 and $F^{x, y}$ are the field strengths of the associated KK gauge fields. ${ }^{16}$

More on the ACS in the two T-duality case. Before proceeding let us discuss the ACS defined in the dual IIB theory in more detail. In general for the case after two Tdualities we find that the ACS is not integrable. Let us illustrate this lack of integrability with the example discussed in section 3.1, which was also used in the discussion of the ACS in the one T-duality case, (4.17) onwards, above.

Starting in the IIB theory with a metric of form $d s^{2}=d z^{i} d \bar{z}^{\bar{i}}$, we now T-dualize along both $x^{1}, y^{1}$. The three holomorphic forms are then given by

$$
\eta_{H}^{1}=\eta^{x}+\tau^{1} \eta^{y}, \quad \eta_{H}^{2}=\eta^{2}+\tau^{2} \eta^{5}, \quad \eta_{H}^{3}=\eta^{3}+\tau^{3} \eta^{6} .
$$

Now considering $d \eta_{H}^{1}$ we find that (4.17) is not met, except at special points in moduli space where $\tau^{1}=\tau^{2}$. Note, this is different from the constraint in moduli space imposed by supersymmetry, (3.4), except at the special point $\tau^{1}=\tau^{2}=i$. Thus, generically, we see that the ACS is not integrable.

\footnotetext{
${ }^{16}$ This follows from the standard relation between the KK gauge potential and the metric, $A_{\mu}^{x}=$ $\hat{j}_{\mu \alpha} \hat{j}^{\alpha x}, \alpha=x, y$, and similarly for $A_{\mu}^{y}$.
} 
One finds that an alternative ACS can be defined which is integrable in this example. It corresponds to keeping the definition of the holomorphic one form $\eta_{H}^{1}, \eta_{H}^{3}$ the same as in (4.30), but changing

$$
\eta_{H}^{2}=\eta^{2}+\tau^{1} \eta^{5}
$$

Now it is easy to see that the integrability condition (4.17) is met.

We have not explored the question of whether an integrable ACS exists in full generality. However, we expect in several cases, obtained for example after two T-dualities, that this is true [46]. If $\hat{\epsilon}$ is the spinor in the dual IIB theory that meets the susy conditions, we write

$$
\hat{\epsilon}=\hat{\epsilon}_{+}+\hat{\epsilon}_{-}
$$

where $\hat{\epsilon}_{ \pm}$are spinors of definite $\mathrm{SO}(6)$ chirality. ${ }^{17}$ An alternate ACS can then be defined, using $\hat{\epsilon}_{+}$, of the form

$$
J_{b}^{a}=\left(\hat{\epsilon}_{+}\right)^{\dagger}\left[\gamma^{a}, \gamma_{b}\right]\left(\hat{\epsilon}_{+}\right)
$$

and similarly, using $\hat{\epsilon}_{-}$. These alternate ACSs are expected to be integrable in several instances.

In the example discussed above for instance, the ACS (4.33) is related to the one used in our susy analysis by a rotation in the $\eta^{2}, \eta^{5}$ plane by $\pi / 2$. This takes $\tau_{2} \rightarrow-1 / \tau_{2}$. In view of the constraint (3.4), this means that the ACS (4.33) in this example is the same as the ACS (4.31), which we argued above is integrable. The definition of the ACS (4.33) is also analogous to that of Strominger [27] (see also [34).

\subsection{Superpotential}

In this section we will first construct a superpotential in the IIA theory obtained by one T-duality in section 4.1 above, which correctly implements the $(2,1)$ condition on the flux $G_{I I A}$. Next we will verify that in the example of section 3.1 the superpotential gives the correct tensions for various BPS branes in the theory, and the correct constraints on moduli. We then briefly present a similar analysis for the IIB theory obtained after two T-dualities (as described in section 4.2). Throughout this subsection, we work in the approximation where the warp factor (2.7) has been set equal to one.

One T-duality: the IIA superpotential. Our discussion in this section makes use of the ACS introduced in section 4.1 (4.11), (4.12). The ACS is parametrized by a period matrix $\tau_{j}^{i}$. One can define a holomorphic $(3,0)$ form (with respect to this ACS), which is given by

$$
\Omega=\eta_{H}^{1} \wedge \eta_{H}^{2} \wedge \eta_{H}^{3}
$$

Note that due to the torus being twisted, $\Omega$ is not closed in general.

The superpotential which implements the $(2,1)$ condition for $G_{I I A}$ is given by

$$
W_{I I A}=\int_{M} G_{\mathrm{IIA}} \wedge \Omega_{\mathrm{IIA}} .
$$

\footnotetext{
${ }^{17}$ Note after two T-dualities the spinor $\hat{\epsilon}$ is not of B-type.
} 
The argument leading to (4.35) is closely analogous to that in the original IIB theory. We begin by noting that $\Omega$ depends on period matrix, $\tau_{j}^{i}$. Varying $\Omega$ with repect to $\tau_{j}^{i}$ gives us a basis of nine $(1,2)$ forms which are well defined on the twisted torus. ${ }^{18}$ Thus demanding that

$$
\partial W_{I I A} / \partial \tau_{j}^{i}=0,
$$

imposes the condition that $G_{I I A}$ has no $(1,2)$ terms in it. ${ }^{19}$

Next, we note that $G_{I I A}$ depends on the field ${ }^{20} \sqrt{g_{x x}} / g_{s}^{I I A}$. Varying with respect to it and demanding that $W_{I I A}=0$ then sets the $(3,0)$ and $(0,3)$ terms of $G_{I I A}$ to vanish, yielding the required result that $G_{I I A}$ is of type $(2,1)$.

One more comment is in order. Notice that $G_{I I A}$ has an explicit dependence on the metric component $g_{x x}$ as well (in the terms proportional to $F_{2}$ etc). However, varying with respect to it does not give any additional constraints. ${ }^{21}$

Brane Tensions in the IIA theory. As described above, the primitive $(2,1)$ ISD condition can be imposed by a superpotential $\int G \wedge \Omega$, supplemented by the condition $J \wedge G=0$. So, we have

$$
W_{\mathrm{IIA}}=\int G_{\mathrm{IIA}} \wedge \Omega_{\mathrm{IIA}},
$$

where $G_{\text {IIA }}$ is given by eq. (4.15). As a check that this is the correct expression, we can compute the tensions of domain walls from wrapped branes, and verify that these tensions satisfy the formula

$$
T_{\text {domain wall }}=a|\Delta W|=a\left|\int \Delta G \wedge \Omega\right|,
$$

with the appropriate choice of $a .^{22}$ For simplicity, we restrict to the case of a $T^{6}$ that factorizes into $T^{2} \times T^{2} \times T^{2}$ with respect to both the Kähler and complex structure moduli:

$$
\begin{aligned}
& d z^{i}=d x^{i}+\tau^{i} d y^{i}, \quad \tau^{i}=\frac{i R_{y^{i}}}{R_{x^{i}}} \quad \text { (no sum) }, \\
& J=\sum \rho_{i} d z^{i} d z^{\bar{\imath}}, \quad \rho_{i}=i R_{x^{i}} R_{y^{i}} \quad \text { (no sum) } .
\end{aligned}
$$

We also assume that the IIB dilaton-axion has no real (axionic) component. It is convenient to define the quantity

$$
\tilde{\Omega}_{\mathrm{IIB}}=R_{x^{1}} R_{x^{2}} R_{x^{3}} \Omega=\left(R_{x^{1}} d x^{1}+i R_{y^{1}} d y^{1}\right) \wedge(1 \rightarrow 2) \wedge(1 \rightarrow 3) .
$$

\footnotetext{
${ }^{18} \mathrm{By}$ well-defined we mean that they have the required periodicity properties imposed by the non-trivial twists. In general, any form which has constant coefficients when expanded in the $\eta$ basis (4.6), (4.7) meets this condition.

${ }^{19}$ We assume here that to begin with $G_{I I A}$ has constant coefficients in the $\eta$ basis. This is manifestly true for the expression (4.15) obtained by T-duality.

${ }^{20}$ This is in fact the dilaton of the original IIB theory rewriten in the IIA variables. It has a partner, which in IIA language is $A_{1(x)}$. Dependence on the partner arises, e.g., from $\tilde{F}_{4}$.

${ }^{21}$ The $\tau_{j}^{x}$ components of the period matrix always come multipled by $g_{x x}$ in $W_{I I A}$.

${ }^{22}$ We will not determine $a$ from first principles here, but it is possible to do so by paying proper attention to normalization. The tension in Einstein frame is then $e^{-\mathcal{K} / 2} \Delta W$, where $\mathcal{K}$ is the Kähler potential. One then needs to convert to string frame to compare to the results of this section.
} 
The utility of this definition is that the components of $\tilde{\Omega}_{\mathrm{IIB}}$ are the powers of $i$ times the volume forms of various three-cycles. In terms of $\tilde{\Omega}_{\mathrm{IIB}}$,

$$
T_{\text {domain wall }}^{\mathrm{IIB}}=c_{\mathrm{IIB}}\left|\int \Delta G_{\mathrm{IIB}} \wedge \tilde{\Omega}_{\mathrm{IIB}}\right|, \quad c_{\mathrm{IIB}}=\frac{a_{\mathrm{IIB}}}{\left(R_{x^{1}} R_{x^{2}} R_{x^{3}}\right)} .
$$

Similarly, in the dual IIA theory obtained by T-dualizing in the $x$-direction, it is convenient to define an analogous quantity $\tilde{\Omega}_{\text {IIA }}$, obtained from (4.41) by replacing $R_{x} d x$ with $R_{x}^{-1} \eta^{x}$. We then obtain a IIA expression for the domain wall tension, of the same form as (4.42). The IIA and IIB expressions for the tension must be equal.

Consider first a D5-brane that wraps a three-cycle $\gamma_{3}$ of $T^{6}$ on IIB side. Then, as we pass through the resulting domain wall in $\mathbb{R}^{3,1}$, we have $\Delta G_{\mathrm{IIB}}=\Delta F_{3}$, where $F_{3} /\left(2 \kappa_{10}^{2} \mu_{D 5}\right)$ is in the cohomology class Poincaré dual to $\gamma_{3}$. Consequently,

$$
c_{\mathrm{IIB}} \int \Delta G_{\mathrm{IIB}} \wedge \tilde{\Omega}_{\mathrm{IIB}}=c_{\mathrm{IIB}} 2 \kappa_{10}^{2} \mu_{\mathrm{D} 5} \int_{\gamma_{3}} \tilde{\Omega}_{\mathrm{IIB}}=c_{\mathrm{IIB}}\left(2 \kappa_{10}^{2} g_{s}^{\mathrm{IIB}}\right) \tau_{\mathrm{D} 5} \int_{\gamma_{3}} \tilde{\Omega}_{\mathrm{IIB}}
$$

Eqs. (4.42) and 4.43) correctly give the tension of the domain wall if

$$
\frac{1}{c_{\mathrm{IIB}}}=2 \kappa_{10}^{2} g_{s}^{\mathrm{IIB}}
$$

and if the pullback of $\tilde{\Omega}_{\text {IIB }}$ to $\gamma_{3}$ is a phase factor times the volume form. It is easy to see from the explicit form of $\tilde{\Omega}$ (4.41), that if the latter is true, then

$$
\begin{aligned}
& \tilde{\Omega}_{\text {IIA }} \wedge R_{x}^{-1} \eta^{x}=e^{i \alpha} \operatorname{Vol}\left(\gamma_{4}\right) \quad\left(\gamma_{4} \text { wrapped by T-dual D6) } \quad \text { D5 } \perp x,\right. \\
& R_{x}\left(\tilde{\Omega}_{\mathrm{IIA}}\right)_{x i j} \frac{1}{2} d x^{i} \wedge d x^{j}=e^{i \alpha} \operatorname{Vol}\left(\gamma_{2}\right) \quad\left(\gamma_{2} \text { wrapped by T-dual D4) D5 } \| x,\right.
\end{aligned}
$$

where $e^{i \alpha}$ is a phase factor and $\operatorname{Vol}(\gamma)$ denotes the volume form on $\gamma$. In these two cases we also have

$$
\begin{aligned}
& g_{x[i} F_{j k]} \frac{1}{3 !} d x^{i} \wedge d x^{j} \wedge d x^{k}=R_{x}^{-2} \eta^{x} \wedge F_{(2)}, \\
& F_{x i j k} \eta^{x} \wedge \frac{1}{3 !} d x^{i} \wedge d x^{j} \wedge d x^{k}=F_{(4)},
\end{aligned}
$$

respectively, so that $G_{\text {IIA }} \wedge \tilde{\Omega}_{\text {IIA }}=R_{x}^{-1} F_{(2)} \wedge \operatorname{Vol}\left(\gamma_{4}\right)$ in the first case, and $G_{\text {IIA }} \wedge \tilde{\Omega}_{\text {IIA }}=$ $R_{x}^{-1} F_{(4)} \wedge \operatorname{Vol}\left(\gamma_{2}\right)$ in the second. Then, from

$$
\frac{1}{2 \kappa_{10}^{2} \mu_{\mathrm{D} 4, \mathrm{D} 6}} \int_{\gamma_{2,4}} F_{(2),(4)}=\mu_{\mathrm{D} 4, \mathrm{D} 6}=\frac{\tau_{\mathrm{D} 4, \mathrm{D} 6}}{g_{s}^{\mathrm{IIA}}},
$$

we have

$$
\begin{aligned}
c_{\mathrm{IIA}} \int \Delta G_{\mathrm{IIA}} \wedge \tilde{\Omega}_{\mathrm{IIA}} & =c_{\mathrm{IIA}} R_{x}^{-1}\left(2 \kappa_{10}^{2} g_{s}^{\mathrm{IIA}}\right) \tau_{\mathrm{D} 4, \mathrm{D} 6} \int_{\gamma_{2,4}} \operatorname{Vol}\left(\gamma_{2,4}\right) \\
& =c_{\mathrm{IIA}}\left(\frac{2 \kappa_{10}^{2} g_{s}^{\mathrm{IIA}}}{R_{x}}\right) T_{\text {domainwall }}^{\mathrm{IIA}}
\end{aligned}
$$


This is a nontrivial consistency check, since we obtain the same proportionality constant

$$
\frac{1}{c_{\mathrm{IIA}}}=\frac{2 \kappa_{10}^{2} g_{s}^{\mathrm{IIA}}}{R_{x}}
$$

in either case.

Similarly, for a wrapped NS5-brane,

$$
\begin{aligned}
& c_{\mathrm{IIB}} \int \Delta G_{\mathrm{IIB}} \wedge \tilde{\Omega}_{\mathrm{IIB}}=c_{\mathrm{IIB}} \frac{i}{g_{s}^{\mathrm{IIB}}} \int \mathcal{H} \wedge \tilde{\Omega}_{\mathrm{IIB}}=c_{\mathrm{IIB}} \frac{i}{g_{s}^{\mathrm{IIB}}}\left(2 \kappa_{10}^{2}\left(g_{s}^{\mathrm{IIB}}\right)^{2}\right) \tau_{\mathrm{NS} 5} \int_{\gamma_{3}} \operatorname{Vol}\left(\gamma_{3}\right) \\
& =c_{\mathrm{IIB}}\left(2 i \kappa_{10}^{2} g_{s}^{\mathrm{IIB}}\right) T_{\text {domain wall }}^{\mathrm{IIB}} \text {. }
\end{aligned}
$$

If the NS5-brane does wrap the $x$-direction, then $H_{i j k}$ in IIA is equal to $\mathcal{H}_{i j k}$ in IIB, and

$$
\begin{aligned}
c_{\mathrm{IIA}} \int \Delta G_{\mathrm{IIA}} \wedge \tilde{\Omega}_{\mathrm{IIA}} & =c_{\mathrm{IIA}} \frac{i}{g_{s}^{\mathrm{IIB}}}\left(2 i \kappa_{10}^{2}\left(g_{s}^{\mathrm{IIA}}\right)^{2}\right) T_{\text {domain wall }}^{\mathrm{IIA}} \\
& =c_{\mathrm{IIA}}\left(2 i \kappa_{10}^{2} g_{s}^{\mathrm{IIA}} R_{x}\right) T_{\text {domain wall }}^{\mathrm{IIA}}
\end{aligned}
$$

where in the last step we have used the T-duality rule $g_{s}^{\mathrm{IIA}}=g_{s}^{\mathrm{IIB}} / R_{x}$.

If the NS5-brane does not wrap the $x$-direction, then it is possible to show by a calculation analogous to (2.19) that

$$
\left(\omega_{(3)}\right)_{i j x}=R_{x}^{-2} \mathcal{H}_{i j x}, \quad\left(\omega_{(3)}\right)_{i j k}=\mathcal{H}_{i j k}=0 \text { otherwise. }
$$

Eqs. (4.53) and (4.54) together give $c_{\mathrm{IIA}}=c_{\mathrm{IIB}} R_{x}^{2}$, which agrees with eqs. (4.51) and (4.44). The carrier of $\left(\omega_{(3)}\right)_{i j x}$ charge is a Kaluza-Klein monopole, which can be regarded as a KK5-brane, or twisted 6-brane [26].

Moduli constraints from the IIA superpotential. In the example considered in section 3.1 it is possible to directly derive the constraints on moduli (3.10) and (3.11) which apply in the T-dual IIA description, by computing and stationarizing the superpotential (4.37). Direct calculation of $G_{\text {IIA }}$ (4.15) using the formulae in appendix $\mathrm{A}$ yields

$$
\begin{aligned}
\frac{1}{2} G_{\mathrm{IIA}}= & d y^{1} \wedge d y^{2} \wedge d y^{3}+\tilde{R}_{x^{1}}^{2}\left(d x^{1}+x^{2} d x^{3}\right) \wedge d x^{2} \wedge d y^{3}- \\
& -\left(\frac{i}{g_{s}^{\mathrm{IIA}}}\right) \tilde{R}_{x^{1}}\left(d y^{1} \wedge d y^{2} \wedge d x^{3}+\tilde{R}_{x^{1}}^{2} d x^{1} \wedge d x^{2} \wedge d x^{3}\right) .
\end{aligned}
$$

So one finds that $W_{\text {IIA }}$ is

$$
\frac{1}{2} W_{\text {IIA }}=1+\rho^{1} \tau^{2}+\left(\frac{\tilde{R}_{x^{1}}}{g_{s}^{\text {IIA }}}\right)\left(\tau^{3}+\rho^{1} \tau^{2} \tau^{3}\right) .
$$

Here the $\rho$ and $\tau$ moduli are being defined as in a conventional square six-torus, so e.g. $\rho^{1}=i \tilde{R}_{x^{1}} R_{y^{1}}$ and so forth. ${ }^{23}$

\footnotetext{
${ }^{23}$ As a reminder, $\tilde{R}_{x^{1}}$ is the radius of the $x^{1}$ circle in the IIA theory. The other radii are the same as in the original IIB theory and are denoted without tildes.
} 
As an aside, we should note that it is not surprising that $1 / g_{s}^{\text {IIA }}$ appears in the superpotential together with a factor of the IIA radius $\tilde{R}_{x^{1}}$. The $10 \mathrm{~d}$ IIB axio-dilaton $\phi$ is a complex scalar, and can appear in a $4 \mathrm{~d} \mathcal{N}=1$ chiral multiplet, and hence in a superpotential. In contrast, the 10d IIA dilaton is a real scalar, and first becomes part of a complex scalar in $9 \mathrm{~d}$; and the combination that appears in $W_{\text {IIA }}$ is precisely (by T-duality) the IIB dilaton $\phi$. This is the field that appears as part of a chiral multiplet in the low-energy $4 \mathrm{~d}$ supersymmetric theory.

At this point, we see immediately that the constraints on moduli that we will find from varying (4.56) will agree with the results (3.10) and (3.11) that follow by dualizing the original IIB constraints. This is because 4.56 precisely agrees (up to the change of notation from IIB to IIA variables) with the original IIB superpotential.

Superpotential after two T-dualities. After two T-dualities, the superpotential is

$$
\hat{W}_{\mathrm{IIB}}=\int \hat{G}_{3} \wedge \hat{\Omega}_{\mathrm{IIB}}
$$

where $\hat{G}_{3}$ is given by eq. (4.28). An argument similar to that of section 4.3 shows that it correctly implements the $(2,1)$ condition on the flux, $\hat{G}_{3}$. A similar identification of domain wall tensions can be performed again in this case. The terms in $\hat{G}_{3}$ and the corresponding domain walls are summarized in the following table:

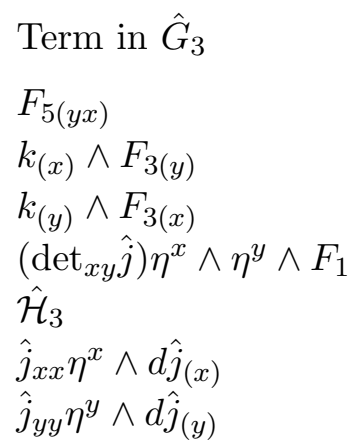

Domain Wall

D3-brane transverse to $x$ and $y$

D5-brane wrapped on $x$, transverse to $y$

D5-brane wrapped on $y$, transverse to $x$

D7-brane wrapped on $x$ and $y$

NS5-brane wrapped on $x$ and $y$

KK5-brane transverse to $x$

KK5-brane transverse to $y$.

\section{Discussion}

The main lesson we can draw from this paper is that new solutions of the string equations of motion based on non Calabi-Yau geometries exist. These can provide supersymmetric vacua after suitable flux is turned on. While we have concentrated on the type-II theories in this paper, it is clear (for example by duality, as in [34) that similar constructions exist in the heterotic theory. Moreover, in the simplest examples described in this paper, we can construct a superpotential sensitive to this departure from "Calabi-Yauness." It would be very interesting to characterize properly in mathematical terms what the class of such compactifications is, and discover how to most efficiently parametrize their moduli spaces and superpotentials. Other recent work in this direction has appeared in [32, 33.

There are also several connections of our new vacua with other backgrounds. As we saw in section 3, in some cases, we expect our models to have a lift to an M-theory compactification on a manifold of special holonomy. It would be very interesting to find 
examples where the M-theory Calabi-Yau (for $\mathcal{N}=2$ ) or $G_{2}($ for $\mathcal{N}=1$ ) geometry could be specified more explicitly. Perhaps one can go further towards determining the Calabi-Yau $X$ which appeared in section 3.1 by gluing together Atiyah-Hitchin and ALE metrics.

Another connection arises by using the duality explored by Hull in [47]. There, it is argued that M-theory on the nilmanifold is dual to the massive type-IIA theory. It follows from this that type-IIA theory compactified on the nilmanifold should be dual to appropriate compactifications of the massive IIA theory. But we have seen in section 3.1 that compactifications of the IIA theory on the nilmanifold (and an additional transverse $T^{3}$ ), with appropriate orientifold actions and background fluxes, yield supersymmetric vacua. This implies that the massive IIA theory, which has no ground state with unbroken supersymmetry in ten dimensions, does have vacua with unbroken supersymmetry in lower dimensions.

There are many clear directions for future work along the lines of this paper:

- We have written down examples of novel geometries which arise by dualizing known vacua. However, the superpotential formulae of section 1 can be applied directly to find supersymmetric vacua. It would be interesting to exhibit a vacuum which is not related by duality to a compactification on a Calabi-Yau orientifold or $G_{2}$ space in any controlled limit.

- Our construction started from the $T^{6} / Z_{2}$ orientifold, and the simplicity of $T^{6}$ is what is responsible for the simple nature of our dual metrics and superpotential formulae. However, it is clear that starting from a more general Calabi-Yau, one could still try to T-dualize along the $T^{3}$ fibers [22]. As long as $H$ does not have any components with more than one leg along the fibers, this should yield a sensible dual geometry. It would be worthwhile to explore the geometries that arise in this way. Some steps in this direction have been taken in the recent papers [32, 33].

- The detailed structure of the moduli spaces of these new compactifications are somewhat mysterious. In the large volume limit, the moduli space should have a description in geometrical terms (analogous to the description of Calabi-Yau moduli space which follows from Yau's theorem). It would be nice to work out an intrinsic formulation of this moduli problem in terms of large radius geometry. In the examples of this paper, and possibly more generally, it could be that the correct procedure is to view all of these vacua as being specified by: i) a choice of Calabi-Yau manifold $M$, ii) a choice of background NS and RR fluxes on $M$, iii) a choice of background "KK monopole flux" or "twisting" on $M$ (in the examples at hand, this corresponds to the choice of twisting in the nilmanifold). One then stationarizes the superpotential (which now contains a contribution from the twisting iii)), and obtains in this way constraints on the "formerly Calabi-Yau" moduli. However this prescription, in addition to being vague, depends on various conjectures (about the existence of metrics with appropriate windings iii) given the Calabi-Yau metric on $M$, for instance) which are not obviously true. 
- We have seen in this paper that compactifications with flux, after T-duality, turn into twisted tori. The twisting gives rise to a non-compact group and the twisted torus can be viewed as a coset of this non-compact group. In the examples studied here these turn out to be generalizations of the nilmanifold. This raises the natural question of whether all Scherk-Schwarz compactifications can be understood in this way as cosets. More generally, the connection between flux, twisted tori and cosets (or orbifolds) needs to be understood better, building on the recent work in [35.

- Finally, it would be nice to see if the new compactifications and computable potentials described here can, together with other ingredients (notable possibilities are D-brane instanton effects and gaugino condensates), lead to models of interest in string cosmology. A discussion of the cosmology arising from IIB Calabi-Yau orientifolds with three-form flux recently appeared in [23], the conclusion being that the potentials obtained to date are too steep to serve a useful role in models of inflation or quintessence.

\section{Acknowledgments}

We would like to acknowledge very useful conversations with A. Dabholkar, S. Gukov, S. Hellerman and G. Moore. We are also grateful to B. Acharya, A. Brandhuber, K. Dasgupta, S. Giddings, J. Gomis, X. Liu, L. McAllister, S. Mukhi, H. Ooguri, S. Prokushkin, T. Ramdas, J. Schwarz, E. Silverstein, H. Verlinde and K. P. Yogendran for helpful comments. S.K. thanks the Aspen Center for Physics for hospitality while this work was in progress. The work of S.K. was supported by a David and Lucile Packard Foundation Fellowship for Science and Engineering, an A.P. Sloan Foundation Fellowship, National Science Foundation grant PHY-0097915, and the DOE under contract DE-AC03-76SF00515. The work of M.S. was supported by the DOE under contract DE-FG03-92-ER40701. S.P.T. acknowledges support from the Swarnajayanti Fellowship, Department of Science and Technology, Government of India. The work of P.K.T. and S.P.T. was supported by the DAE. Most of all, P.K.T. and S.P.T. thank the people of India.

\section{A. T-duality maps of fields}

In this appendix we express the T-duality maps from IIA to IIB and vice versa. We begin by first explaining our conventions and notation. We will mainly use the conventions of Polchinski [48] for the supergravity fields (with the same normalisations). In particular,

$$
\tilde{F}_{4}=d C_{3}+A_{1} \wedge H_{3}, \quad \tilde{F}_{5}=d C_{4}-\frac{1}{2} C_{2} \wedge \mathcal{H}_{3}+\frac{1}{2} \mathcal{B}_{2} \wedge F_{3},
$$

stand for the gauge invariant RR 4-form and 5-form field strengths in the IIA and IIB theories. Also we will use the notation

$$
\tilde{F}_{3}=F_{3}-C_{0} \mathcal{H}_{3},
$$

in the IIB theory. 
We also remind the reader of some of our notation. $x$ denotes the direction along which we T-dualise. $\mu, \nu$ denote corrdinate indices and take six values, $\alpha, \beta$ denote all coordinate indices except $x$ and take five values. Finally, $a, b$, denote tangent space indices.

To save clutter we denote the metric as $j_{\mu \nu}$ and $g_{\mu \nu}$ in the IIB and IIA theories respectively. We also denote the NS two form potential and field strengths as $\mathcal{B}, \mathcal{H}_{3}$ and $B, H_{3}$ in the IIB and IIA theories respectively.

Given an $n$ form $F_{n}$ we use the notation $F_{n(x)}$ to denote an $n-1$ form whose components are given by:

$$
\left[F_{n(x)}\right]_{i_{1}, \cdots i_{n-1}}=\left[F_{n}\right]_{x i_{1} \cdots i_{n-1}}
$$

Finally

$$
j_{(x)}=\frac{1}{j_{x x}} j_{x \alpha} d x^{\alpha}, \quad g_{(x)}=\frac{1}{g_{x x}} g_{x \alpha} d x^{\alpha}
$$

denote one forms in IIB and IIA theory and

$$
\omega_{(x)}=-d g_{(x)}
$$

denotes a two form in IIA theory.

With these definitions, the map between IIA, and IIB theory under T-duality in the $x$-direction is given as follows 43, 44: the Neveu-Schwarz fields transform as

$$
\begin{aligned}
g_{x x} & =\frac{1}{j_{x x}} \\
g_{x \alpha} & =-\frac{\mathcal{B}_{x \alpha}}{j_{x x}} \\
g_{\alpha \beta} & =j_{\alpha \beta}-\frac{1}{j_{x x}}\left(j_{x \alpha} j_{x \beta}-\mathcal{B}_{x \alpha} \mathcal{B}_{x \beta}\right) \\
B_{x \alpha} & =-\frac{j_{x \alpha}}{j_{x x}} \\
B_{\alpha \beta} & =\mathcal{B}_{\alpha \beta}-\frac{1}{j_{x x}}\left(j_{x \alpha} \mathcal{B}_{x \beta}-\mathcal{B}_{x \alpha} j_{x \beta}\right) \\
g_{s}^{I I A} & =\frac{g_{s}^{I I B}}{\sqrt{j_{x x}}}
\end{aligned}
$$

Here the left hand refers to fields in the IIA theory and the right hand side to fields in the IIB theory. For the three form field strength, $H_{3}$ this takes the form,

$$
\begin{aligned}
H_{3(x)} & =d j_{(x)} \\
H_{3} & =\mathcal{H}_{3}-\mathcal{H}_{3(x)} \wedge j_{(x)}-\mathcal{B}_{(x)} \wedge d j_{(x)} .
\end{aligned}
$$

The Ramond fields transform as

$$
\begin{aligned}
F_{2(x)} & =F_{1} \\
F_{2} & =\tilde{F}_{3(x)}-\mathcal{B}_{(x)} \wedge F_{1} \\
\tilde{F}_{4(x)} & =\tilde{F}_{3}-j_{(x)} \wedge \tilde{F}_{3(x)} \\
\tilde{F}_{4} & =\tilde{F}_{5(x)}-\mathcal{B}_{(x)} \wedge\left(\tilde{F}_{3}-j_{(x)} \wedge \tilde{F}_{3(x)}\right) .
\end{aligned}
$$


In the formulae above, a field strength with and without a leg along the $x$ direction are denoted as $F_{n(x)}$, and $F_{n}$ respectively.

The inverse of these expressions is given by

$$
\begin{aligned}
j_{x x} & =\frac{1}{g_{x x}} \\
j_{x \alpha} & =-\frac{B_{x \alpha}}{g_{x x}} \\
j_{\alpha \beta} & =g_{\alpha \beta}-\frac{1}{g_{x x}}\left(g_{x \alpha} g_{x \beta}-B_{x \alpha} B_{x \beta}\right) \\
\mathcal{B}_{x \alpha} & =-\frac{g_{x \alpha}}{g_{x x}} \\
\mathcal{B}_{\alpha \beta} & =B_{\alpha \beta}-\frac{1}{g_{x x}}\left(g_{x \alpha} B_{x \beta}-B_{x \alpha} g_{x \beta}\right) \\
g_{s}^{I I B} & =\frac{g_{s}^{I I A}}{\sqrt{g_{x x}}} \\
\mathcal{H}_{3(x)} & =-\omega_{(x)} \\
\mathcal{H}_{3} & =H_{3}+\omega_{(x)} \wedge B_{(x)}-g_{(x)} \wedge H_{3(x)}
\end{aligned}
$$

and

$$
\begin{aligned}
F_{1} & =F_{2(x)} \\
\tilde{F}_{3(x)} & =F_{2}-g_{x} \wedge F_{2(x)} \\
\tilde{F}_{3} & =\tilde{F}_{4(x)}-B_{(x)} \wedge\left(F_{2}-g_{x} \wedge F_{2(x)}\right) \\
\tilde{F}_{5(x)} & =\tilde{F}_{4}-g_{(x)} \wedge \tilde{F}_{4(x)} .
\end{aligned}
$$

\section{B. T-duality map of spinor conditions}

In this appendix we discuss the T-duality transformation rules for the IIB and IIA spinor conditions. Our discussion largely follows [44. We first discuss the map for T-duality along one direction and then, more briefly, two directions.

One T-duality. We denote the spinors and vielbein in the IIB (IIA) theory with (without) a tilde superscript. The vielbein in IIB and IIA theory are related by

$$
\tilde{e}_{a}^{\mu}=Q_{\nu}^{\mu} e_{a}^{\nu}
$$

where the matrix $Q$ is given by

$$
Q=\left(\begin{array}{cc}
g_{x x} & (g+B)_{x \alpha} \\
0 & \mathbf{1}
\end{array}\right)
$$

Gamma matrices with tangent space indices are the same in the IIB and IIA theories, i.e.

$$
\tilde{\gamma}^{a}=\gamma^{a}
$$

We will denote these as $\gamma^{a}$ below. 
One can show that if

$$
\tilde{\epsilon}=\tilde{\epsilon}_{L}+i \tilde{\epsilon}_{R},
$$

meets the spinor conditions imposed by susy in the IIB theory, then

$$
\epsilon=\epsilon_{L}+i \epsilon_{R}
$$

meets the spinor conditions in the IIA theory. Here

$$
\begin{aligned}
& \tilde{\epsilon}_{L}=\Omega \epsilon_{L}, \\
& \tilde{\epsilon}_{R}=\epsilon_{R},
\end{aligned}
$$

and

$$
\Omega=-\frac{1}{\sqrt{g_{x x}}} \gamma_{11} \gamma_{9}
$$

The above statement is true if the IIB and IIA fields are related under T-duality by the transformations (A.6), (A.10).

We now turn to mapping the spinor conditions of type-IIB to type-IIA. We will not demonstrate the map in full detail. Instead we give one representative example. Consider the dilatino variation in the IIB theory. Since the dilaton-axion is constant in the IIB theory, it is (eq. (2.3) of [42]):

$$
\delta \tilde{\lambda}=-\frac{i}{24} \tilde{G} \tilde{\epsilon}
$$

where

$$
\tilde{G}=\tilde{\gamma}^{\mu \nu \sigma}\left[G_{3}\right]_{\mu \nu \sigma}
$$

with

$$
\left[G_{3}\right]_{\mu \nu \sigma} \equiv\left[F_{3}\right]_{\mu \nu \sigma}-\phi\left[\mathcal{H}_{3}\right]_{\mu \nu \sigma}=\left[\tilde{F}_{3}\right]_{\mu \nu \sigma}-\left(\frac{i}{g_{s}^{I I B}}\right)\left[\mathcal{H}_{3}\right]_{\mu \nu \sigma}
$$

and $\tilde{\epsilon}=\tilde{\epsilon}_{L}+i \tilde{\epsilon}_{R}$. Then using the relation between the vierbeins (B.1), we have the relations:

$$
\tilde{\gamma}^{x}=g_{x x} \gamma^{x}+[g+B]_{x \alpha} \gamma^{\alpha}
$$

and

$$
\tilde{\gamma}^{\alpha}=\gamma^{\alpha} \text {. }
$$

Using the T-duality relations between the fields (A.10), the flux

$$
\begin{array}{r}
{\left[\mathcal{H}_{3}\right]_{\alpha \beta \gamma}=\left[H_{3}\right]_{\alpha \beta \gamma}+\left[\omega_{(x)} \wedge B_{(x)}\right]_{\alpha \beta \gamma}-\left[g_{(x)} \wedge H_{3(x)}\right]_{\alpha \beta \gamma}} \\
{\left[\mathcal{H}_{3}\right]_{x \alpha \beta}=-\omega_{x \alpha \beta} .}
\end{array}
$$

So

$$
\tilde{\gamma}^{\mu \nu \rho}\left[\mathcal{H}_{3}\right]_{\mu \nu \rho}=\left[H_{3}-g_{x x} g_{(x)} \wedge \omega_{(x)}-g_{(x)} \wedge H_{3(x)}\right]_{\alpha \beta \gamma} \gamma^{\alpha \beta \gamma}-g_{x x} \gamma^{x \alpha \beta} \omega_{x \alpha \beta} .
$$

As mentioned at the end of this appendix, the r.h.s. can be written as follows:

$$
\tilde{\gamma}^{\mu \nu \rho}\left[\mathcal{H}_{3}\right]_{\mu \nu \rho}=\left[H_{3}-g_{x x} \eta^{x} \wedge \omega_{(x)}-\eta^{x} \wedge H_{3(x)}\right]_{\mu \nu \rho} \gamma^{\mu \nu \rho} .
$$


Similarly, using (A.10),

$$
\left[\tilde{F}_{3}\right]_{\alpha \beta \gamma}=\left[\tilde{F}_{4}\right]_{x \alpha \beta \gamma}-\left[B_{(x)} \wedge F_{2}\right]_{\alpha \beta \gamma}+\left[B_{(x)} \wedge g_{(x)} \wedge F_{2(x)}\right]_{\alpha \beta \gamma}
$$

and

$$
\left[\tilde{F}_{3}\right]_{x \alpha \beta}=\left[F_{2}-g_{(x)} \wedge F_{2(x)}\right]_{\alpha \beta}
$$

So we find

$$
\tilde{\gamma}^{\mu \nu \rho}\left[\tilde{F}_{3}\right]_{\mu \nu \rho}=\left[\tilde{F}_{4(x)}+g_{x x} g_{(x)} \wedge F_{2}\right]_{\alpha \beta \gamma} \gamma^{\alpha \beta \gamma}+g_{x x}\left[F_{2}-g_{(x)} \wedge F_{2(x)}\right]_{\alpha \beta} \gamma^{x \alpha \beta} .
$$

Once again as discussed at the end of the appendix this can be expressed as

$$
\tilde{\gamma}^{\mu \nu \rho}\left[\tilde{F}_{3}\right]_{\mu \nu \rho}=\left[\tilde{F}_{4(x)}+g_{x x} \eta^{x} \wedge F_{2}\right]_{\mu \nu \rho} \gamma^{\mu \nu \rho} .
$$

From (B.15), (B.17) we obtain

$$
\tilde{\gamma}^{\mu \nu \sigma}\left[G_{3}\right]_{\mu \nu \sigma}=\gamma^{\mu \nu \sigma}\left[G_{I I A}\right]_{\mu \nu \sigma},
$$

where $G_{\text {IIA }}$ is a three-form in the IIA theory given by

$$
G_{\mathrm{IIA}}=\left(\tilde{F}_{4(x)}+g_{x x} \eta^{x} \wedge F_{2}\right)-\left(\frac{i \sqrt{g}}{g_{s x}^{I I A}}\right)\left(H_{3}-g_{x x} \eta^{x} \wedge w_{(x)}-\eta^{x} \wedge H_{3(x)}\right) .
$$

Setting the dilatino variation to zero in IIB sets the r.h.s. of (B.18) to zero, yielding the condition that

$$
\gamma^{\mu \nu \sigma}\left[G_{I I A}\right]_{\mu \nu \sigma} \tilde{\epsilon}=0 .
$$

The ACS in IIA is defined by the relation (4.11). In turn this defines a holomorphic basis, (4.12), in which

$$
\gamma^{\bar{\imath}} \tilde{\epsilon}=0
$$

It is then easy to see that $\mathrm{B} .20$ leads to the condition that the term in $G_{I I A}$ of type $(3,0)$ vanish and that the $(2,1)$ term satsifies the condition of primitivity $(4.16)$.

It is straightforward to repeat this discussion for the gravitino variation in IIB theory. Mapping the resulting conditions to IIA yield the additional constraints that $G_{I I A}$ is purely of type $(2,1)$ and primitive.

We end this discussion of the one T-duality case with two observations. First, we note that (B.15) follows from (B.14), and (B.17) follows from (B.16), due to the fact that

$$
\left[\omega_{x} \wedge d x\right]_{\alpha \beta \gamma}=\left[H_{3(x)} \wedge d x\right]_{\alpha \beta \gamma}=\left[g_{(x)} \wedge H_{3(x)}\right]_{x \alpha \beta}=\left[g_{(x)} \wedge \omega_{(x)}\right]_{x \alpha \beta}=0 .
$$

Second, we note that the last term in the expression (B.19) for $G_{\text {IIA }}$ is absent. It arises from the term $d j_{(x)}$ in the original IIB theory on $T^{6} / Z_{2}$, which vanishes since $j_{(x)}$ is a constant in that theory. Thus we have (4.15). 
Two T-dualities A similar analysis can be carried out for T-duality along two directions. Here we state the main conclusions in obvious notation.

One finds that ${ }^{24}$

$$
T_{y} \circ T_{x}: \quad\left[\mathcal{H}_{3}\right]_{\mu \nu \rho} \tilde{\gamma}^{\mu \nu \rho}=\left[\hat{H}_{3}\right]_{\hat{\alpha} \hat{\beta} \hat{\gamma}} \gamma^{\hat{\alpha} \hat{\beta} \hat{\gamma}}+\left[\hat{j}_{x x} \eta^{x} \wedge d \hat{j}_{(x)}+\hat{j}_{y y} \eta^{y} \wedge d \hat{j}_{(y)}\right]_{\mu \nu \rho} \hat{\gamma}^{\mu \nu \rho}
$$

and for the Ramond-Ramond field strength

$$
\begin{gathered}
T_{y} \circ T_{x}: \quad\left[\tilde{F}_{3}\right]_{\mu \nu \rho} \tilde{\gamma}^{\mu \nu \rho}=[ \\
{\left[\tilde{F}_{5(y x)}+\left(\hat{j}_{x x} \eta^{x}+\hat{j}_{x y} \eta^{y}\right) \wedge \hat{\tilde{F}}_{3(y)}-\left(\hat{j}_{x y} \eta^{x}+\hat{j}_{y y} \eta^{y}\right) \wedge \hat{\tilde{F}}_{3(x)}+\right.} \\
\left.+\operatorname{det}_{x y} \hat{j} \eta^{x} \wedge \eta^{y} \wedge F_{1}\right]_{\mu \nu \rho} \gamma^{\mu \nu \rho}
\end{gathered}
$$

Here the indices $\hat{\alpha}, \hat{\beta}, \hat{\gamma}$ run over the compact directions except the T dualized ones $x$ and $y$. The above expressions imply that

$$
T_{y} \circ T_{x}: \quad\left[G_{3}\right]_{\mu \nu \rho} \tilde{\gamma}^{\mu \nu \rho}=\left[\hat{G}_{3}\right]_{\mu \nu \rho} \hat{\gamma}^{\mu \nu \rho}
$$

where

$$
\begin{aligned}
\hat{G}_{3}=\{ & \hat{\tilde{F}}_{5(y x)}+\left(\hat{j}_{x x} \eta^{x}+\hat{j}_{x y} \eta^{y}\right) \wedge \hat{\tilde{F}}_{3(y)}-\left(\hat{j}_{x y} \eta^{x}+\hat{j}_{y y} \eta^{y}\right) \wedge \hat{\tilde{F}}_{3(x)}+ \\
& \left.+\operatorname{det}_{x y} \hat{j} \eta^{x} \wedge \eta^{y} \wedge \hat{F}_{1}\right\}-\left(\frac{i}{g_{s}^{I I B}}\right) \sqrt{\operatorname{det}_{x y} \hat{j}}\left\{\hat{\mathcal{H}}_{3}+\hat{j}_{x x} \eta^{x} \wedge d \hat{j}_{(x)}+\hat{j}_{y y} \eta^{y} \wedge d \hat{j}_{(y)}\right\} .
\end{aligned}
$$

Here we have used the definitions

$$
\begin{array}{rlrl}
{\left[\hat{\tilde{F}}_{5(y x)}\right]_{\hat{\alpha} \hat{\beta} \hat{\gamma}}} & =\left[\hat{\tilde{F}}_{5}\right]_{y x \hat{\alpha} \hat{\beta} \hat{\gamma}} & \\
{\left[\hat{\tilde{F}}_{3(x)}\right]_{\hat{\alpha} \hat{\beta}}} & =\left[\hat{\tilde{F}}_{3}\right]_{x \hat{\alpha} \hat{\beta}}, & & {\left[\hat{\tilde{F}}_{3(y)}\right]_{\hat{\alpha} \hat{\beta}}=\left[\hat{\tilde{F}}_{3}\right]_{y \hat{\alpha} \hat{\beta}}} \\
{\left[\hat{H}_{3(x)}\right]_{\hat{\alpha} \hat{\beta}}} & =\left[\hat{H}_{3}\right]_{x \hat{\alpha} \hat{\beta}}, & & {\left[\hat{H}_{(y)}\right]_{\hat{\alpha} \hat{\beta}}=\left[\hat{H}_{3}\right]_{y \hat{\alpha} \hat{\beta}}}
\end{array}
$$

and

$$
\operatorname{det}_{x y} \hat{j}=\left(\hat{j}_{x x} \hat{j}_{y y}-\hat{j}_{x y}^{2}\right), \quad \hat{j}_{(x)}=\frac{\hat{j}_{x \hat{\alpha}} d x^{\hat{\alpha}}}{\hat{j}_{x x}}, \quad \hat{j}_{(y)}=\frac{\hat{j}_{y \hat{\alpha}} d x^{\hat{\alpha}}}{\hat{j}_{y y}}
$$

Now, in analogy with the one T-duality case, the choice of ACS discussed in section 4.2 before eq. (4.27) defines a basis of holomorphic one forms. In this basis one can then conclude that $\hat{G}$ is of type $(2,1)$ and primitive.

\section{References}

[1] P. Candelas, G.T. Horowitz, A. Strominger and E. Witten, Vacuum configurations for superstrings, Nucl. Phys. B 258 (1985) 46.

[2] Mirror Symmetry I, S.T. Yau ed., American RI/Cambridge, MA, AMS/International Press, Providence 1998.

\footnotetext{
${ }^{24}$ Terms proportional to $\hat{H}_{3(x)}, \hat{H}_{3(y)}$ are absent due to the orientifold projection.
} 
[3] J. Lykken, E. Poppitz and S.P. Trivedi, Branes with guts and supersymmetry breaking, Nucl. Phys. B 543 (1999) 105 hep-th/9806080;

G. Aldazabal, S. Franco, L.E. Ibáñez, R. Rabadán and A.M. Uranga, $D=4$ chiral string compactifications from intersecting branes, J. Math. Phys. 42 (2001) 3103 [hep-th/0011073; S.D. Joglekar and B.P. Mandal, Application of finite field-dependent brs transformations to problems of the coulomb gauge, Int. J. Mod. Phys. A 17 (2002) 1279 hep-th/0105042;

M. Cvetič, G. Shiu and A.M. Uranga, Three-family supersymmetric standard like models from intersecting brane worlds, Phys. Rev. Lett. 87 (2001) 201801 hep-th/0107143;

D. Cremades, L. Ibanez and F. Marchesano, Standard model at intersecting D5-branes: lowering the string scale, Nucl. Phys. B 643 (2002) 93 hep-th/0205074;

R. Blumenhagen, V. Braun, B. Körs and D. Lüst, Orientifolds of K3 and Calabi-Yau manifolds with intersecting D-branes, J. High Energy Phys. 07 (2002) 026 [hep-th/0206038;

A.M. Uranga, Local models for intersecting brane worlds, J. High Energy Phys. 12 (2002) 058 hep-th/0208014;

C. Kokorelis, Exact standard model structures from intersecting branes, hep-th/0210004:

D. Bailin, G.V. Kraniotis and A. Love, Standard-like models from intersecting D5-branes, Phys. Lett. B 553 (2003) 79 hep-th/0210219;

R. Blumenhagen, L. Görlich and T. Ott, Supersymmetric intersecting branes on the type-IIA $T^{6} / \mathbb{Z}_{4}$ orientifold, J. High Energy Phys. 01 (2003) 021 hep-th/0211059.

[4] S. Gukov, C. Vafa and E. Witten, CFT's from Calabi-Yau four-folds, Nucl. Phys. B 584 (2000) 69 hep-th/9906070.

[5] K. Dasgupta, G. Rajesh and S. Sethi, M-theory, orientifolds and G-flux, J. High Energy Phys. 08 (1999) 023 hep-th/9908088.

[6] T.R. Taylor and C. Vafa, RR flux on Calabi-Yau and partial supersymmetry breaking, Phys. Lett. B 474 (2000) 130 hep-th/9912152.

[7] G. Curio, A. Klemm, D. Lüst and S. Theisen, On the vacuum structure of type-II string compactifications on Calabi-Yau spaces with $H$ fluxes, Nucl. Phys. B 609 (2001) 3 hep-th/0012213.

[8] M. Haack and J. Louis, M-theory compactified on Calabi-Yau fourfolds with background flux, Phys. Lett. B 507 (2001) 296 hep-th/0103068.

[9] S.B. Giddings, S. Kachru and J. Polchinski, Hierarchies from fluxes in string compactifications, Phys. Rev. D 66 (2002) 106006 hep-th/0105097.

[10] E. Silverstein, (A)dS backgrounds from asymmetric orientifolds, hep-th/0106209.

[11] K. Becker and M. Becker, Supersymmetry breaking, M-theory and fluxes, J. High Energy Phys. 07 (2001) 038 hep-th/0107044.

[12] S. Kachru, M.B. Schulz and S. Trivedi, Moduli stabilization from fluxes in a simple IIB orientifold, hep-th/0201028.

[13] A.R. Frey and J. Polchinski, $N=3$ warped compactifications, Phys. Rev. D 65 (2002) 126009 hep-th/0201029.

[14] J. Louis and A. Micu, Type-II theories compactified on Calabi-Yau threefolds in the presence of background fluxes, Nucl. Phys. B 635 (2002) 395 hep-th/0202168.

[15] R. Argurio, V.L. Campos, G. Ferretti and R. Heise, Freezing of moduli with fluxes in three dimensions, Nucl. Phys. B 640 (2002) 351 hep-th/0205295. 
[16] K. Becker and M. Becker, M-theory on eight-manifolds, Nucl. Phys. B 477 (1996) 155 hep-th/9605053.

[17] B. Greene, K. Schalm and G. Shiu, Warped compactifications in $M$ and $F$ theory, Nucl. Phys. B $584(2000) 480$ hep-th/0004103.

[18] H. Verlinde, Holography and compactification, Nucl. Phys. B 580 (2000) 264 hep-th/9906182;

C.S. Chan, P.L. Paul and H. Verlinde, A note on warped string compactification, Nucl. Phys. B 581 (2000) 156 hep-th/0003236.

[19] P. Mayr, On supersymmetry breaking in string theory and its realization in brane worlds, Nucl. Phys. B 593 (2001) 99 [hep-th/0003198; Stringy world branes and exponential hierarchies, J. High Energy Phys. 11 (2000) 013 hep-th/0006204.

[20] O. DeWolfe and S.B. Giddings, Scales and hierarchies in warped compactifications and brane worlds, hep-th/0208123.

[21] L. Randall and R. Sundrum, A large mass hierarchy from a small extra dimension, Phys. Rev. Lett. 83 (1999) 3370 hep-ph/9905221.

[22] A. Strominger, S.-T. Yau and E. Zaslow, Mirror symmetry is T-duality, Nucl. Phys. B 479 (1996) 243 hep-th/9606040.

[23] A.R. Frey and A. Mazumdar, 3-form induced potentials, dilaton stabilization and running moduli, Phys. Rev. D 67 (2003) 046006 hep-th/0210254;

S. Kachru, R. Kallosh and S. Trivedi, unpublished.

[24] T.H. Buscher, A symmetry of the string background field equations, Phys. Lett. B 194 (1987) 59; Path integral derivation of quantum duality in nonlinear sigma models, Phys. Lett. B 201 (1988) 466.

[25] J. Scherk and J.H. Schwarz, How to get masses from extra dimensions, Nucl. Phys. B 153 (1979) 61.

[26] C.M. Hull, Gravitational duality, branes and charges, Nucl. Phys. B 509 (1998) 216 hep-th/9705162.

[27] A. Strominger, Superstrings with torsion, Nucl. Phys. B 274 (1986) 253.

[28] S. Gukov, Solitons, superpotentials and calibrations, Nucl. Phys. B 574 (2000) 169 hep-th/9911011.

[29] S. Ferrara and M. Porrati, $N=1$ no scale supergravity from IIB orientifolds, Phys. Lett. B $\mathbf{5 4 5}(2002) 411$ hep-th/0207135.

[30] R. D'Auria, S. Ferrara and S. Vaula, $N=4$ gauged supergravity and a IIB orientifold with fluxes, New J. Phys. 4 (2002) 71 hep-th/0206241.

[31] L. Andrianopoli, R. D'Auria, S. Ferrara and M.A. Lledo, Gauging of flat groups in four dimensional supergravity, J. High Energy Phys. 07 (2002) 010 hep-th/0203206.

[32] S. Gurrieri, J. Louis, A. Micu and D. Waldram, Mirror symmetry in generalized Calabi-Yau compactifications, Nucl. Phys. B 654 (2003) 61 hep-th/0211102.

[33] G.L. Cardoso et al., Non-Kähler string backgrounds and their five torsion classes, Nucl. Phys. B $652(2003) 5$ hep-th/0211118. 
[34] K. Becker and K. Dasgupta, Heterotic strings with torsion, J. High Energy Phys. 11 (2002) 006 hep-th/0209077;

K. Becker, M. Becker, K. Dasgupta and P. Green, to appear.

[35] A. Dabholkar and C. Hull, Duality twists, orbifolds, and fluxes, hep-th/0210209.

[36] S. Hellerman, J. McGreevy and B. Williams, Geometric constructions of nongeometric string theories, hep-th/0208174.

[37] S. Gukov, private discussions.

[38] N. Kaloper and R.C. Myers, The $O(d d)$ story of massive supergravity, J. High Energy Phys. 05 (1999) 010 hep-th/9901045.

[39] J. Shin, Group actions on the 3-dimensional nilmanifold, Trends in Mathematics 1 (1998) 62.

[40] S. Kachru, X. Liu, M. Schulz and S.P. Trivedi, Supersymmetry changing bubbles in string theory, hep-th/0205108.

[41] A. Sen, A note on enhanced gauge symmetries in $M$ - and string theory, J. High Energy Phys. 09 (1997) 001 hep-th/9707123.

[42] M. Grana and J. Polchinski, Gauge/gravity duals with holomorphic dilaton, Phys. Rev. D 65 (2002) 126005 hep-th/0106014.

[43] E. Bergshoeff, C.M. Hull and T. Ortin, Duality in the type-II superstring effective action, Nucl. Phys. B 451 (1995) 547 hep-th/9504081.

[44] S.F. Hassan, T-duality, space-time spinors and RR fields in curved backgrounds, Nucl. Phys. B 568 (2000) 145 hep-th/9907152.

[45] M. Nakahara, Geometry, topology and physics, Institute of Physics Publishing, Bristol 1990.

[46] P.K. Tripathy and S.P. Trivedi, Compactifications with flux on K3 and tori, to appear.

[47] C.M. Hull, Massive string theories from M-theory and F-theory, J. High Energy Phys. 11 (1998) 027 hep-th/9811021.

[48] J. Polchinski, String theory, vol. II, Cambridge University Press, Cambidge 1998. 